Article

\title{
Asymmetric 1,4-Michael Addition Reaction of Azadienes with $\alpha$-Thiocyanoindanones Catalyzed by Bifunctional Chiral Squaramide
}

\author{
Xiao-Yan Dong and Da-Ming Du *(D) \\ School of Chemistry and Chemical Engineering, Beijing Institute of Technology, Beijing 100081, China; \\ 18332766507@163.com \\ * Correspondence: dudm@bit.edu.cn; Tel.: +86-106-891-4985
}

check for

updates

Citation: Dong, X.-Y.; Du, D.-M.

Asymmetric 1,4-Michael Addition

Reaction of Azadienes with

$\alpha$-Thiocyanoindanones Catalyzed by Bifunctional Chiral Squaramide.

Molecules 2021, 26, 5146. https://

doi.org/10.3390/molecules26175146

Academic Editor: Rafael Chinchilla

Received: 3 August 2021

Accepted: 22 August 2021

Published: 25 August 2021

Publisher's Note: MDPI stays neutral with regard to jurisdictional claims in published maps and institutional affiliations.

Copyright: (C) 2021 by the authors. Licensee MDPI, Basel, Switzerland. This article is an open access article distributed under the terms and conditions of the Creative Commons Attribution (CC BY) license (https:/ / creativecommons.org/licenses/by/ $4.0 /)$.

\begin{abstract}
In this paper, the organocatalytic asymmetric 1,4-Michael addition reaction of azadienes and $\alpha$-thiocyanoindanones was investigated. A series of chiral benzofuran compounds containing thiocyano group and quaternary carbon center were synthesized in moderate yields with good enantioselectivities (up to 90:10 er ) and high diastereoselectivities (up to $>95: 5 d r$ ). This is the first case of 1,4-Michael addition reaction using $\alpha$-thiocyanoindanones to obtain a series of chiral thiocyano compounds and further broaden the scope of application of azadiene substrates. In addition, a possible reaction mechanism is also described in the article.
\end{abstract}

Keywords: asymmetric catalysis; organocatalysis; 1,4-Michael addition; azadiene; benzofuran

\section{Introduction}

Benzofuran derivatives are an important class of heterocyclic compounds in many biologically active natural products [1-4]. Compounds containing benzofuran structural motifs exhibit excellent anticancer, antioxidation, and antifungal activities, and they can also be used as important structural motifs for some organic materials and drug molecules (Figure 1) [5-8]. The development of efficient and convenient ways to obtain compounds containing benzofuran structural motifs is the common goal pursued by many organic chemists. As far as we know, azadienes derived from benzofurans can undergo 1,4Michael addition reactions with suitable nucleophiles due to their high reactivity to quickly construct multisubstituted benzofuran derivatives, and a variety of benzofuran derivatives have been successfully obtained by using azadienes [9-14].

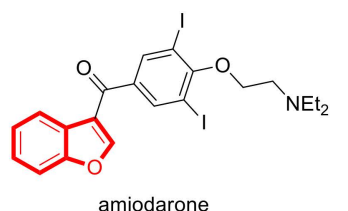

amiodarone

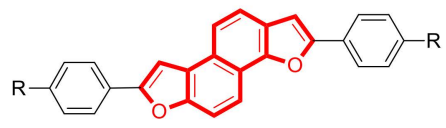

organic transistor
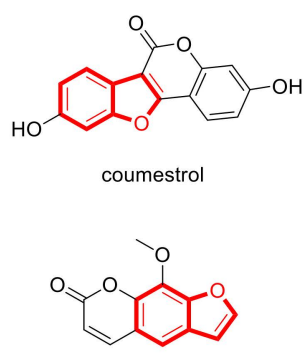

methoxsalen
Figure 1. Selected biologically active benzofuran derivatives.

In addition, thiocyanate compounds have various biological activities such as being anticancer, antimicrobial, and insecticidal; they have a wide range of applications in the fields of medicine, pesticides, and materials (Figure 2). Thiocyanate compounds are also important synthetic intermediates for obtaining sulfur-containing compounds [15-20]. 
Therefore, thiocyanate compounds have attracted the attention of more and more organic chemists. As far as we know, thiocyanate or trimethylsilyl alkyl isothiocyanates can react with substrates with leaving groups to obtain thiocyano compounds. Mainly include ${ }^{-} \mathrm{SCN}$ nucleophilic substitution reaction, ${ }^{+} \mathrm{SCN}$ electrophilic substitution reaction, and $\bullet \mathrm{SCN}$ radical reaction. In addition, the reaction of cyanating reagents with sulfur-containing substrates is also an important method for obtaining thiocyanate compounds. Mainly include ${ }^{-} \mathrm{CN}$ nucleophilic substitution reaction, ${ }^{+} \mathrm{CN}$ electrophilic substitution reaction, and $\bullet \mathrm{CN}$ radical reaction [21-26]. In recent years, the application research of thiocyanate compounds has also developed rapidly. However, in the development process, it also faces problems such as a low utilization rate of substrate atoms and poor economy $[27,28]$. $\alpha-$ Thiocyanoindanone is a nucleophile with a thiocyanato functional group; notably, by using $\alpha$-thiocyanoindanone as a nucleophile, thiocyanate compounds can be obtained efficiently and conveniently. However, at present, the development of $\alpha$-thiocyanoindanone in the asymmetric field is still relatively limited. To the best of our knowledge, there are few reports on the asymmetric catalytic reaction of $\alpha$-thiocyanoindanones. In 2017, Yu and coworkers used $\alpha$-thiocyanoindanones and $\alpha$-aminosulfones to undergo a Mannich reaction, which obtained a series of optically active 2-thiocyanato-2-(1-aminoalkyl)-substituted 1-tetralones and 1-indanones (Scheme 1a) [29]. Regrettably, there has been no report about a series of optically active thiocyanate compounds by using $\alpha$-thiocyanoindanone as a Michael donor to undergo a 1,4-addition asymmetric reaction.

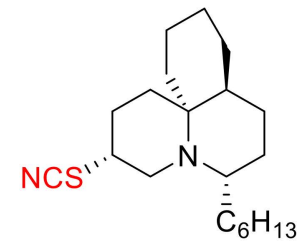<smiles>CC(C)C1CC2(C)CCC1(C)C2(C#N)C(C)C</smiles>

9-thiocyanato pupukeanane<smiles></smiles>

cylindricine $\mathrm{F}\left(\mathrm{R}=\mathrm{C}_{6} \mathrm{H}_{13}\right)$ cylindricine $\mathrm{G}\left(\mathrm{R}=\mathrm{C}_{4} \mathrm{H}_{9}\right)$<smiles>N#[Si]CCOc1ccc(Oc2ccccc2)cc1</smiles>

Figure 2. Selected biologically active molecules containing thiocyano group.

(a) Yu's work

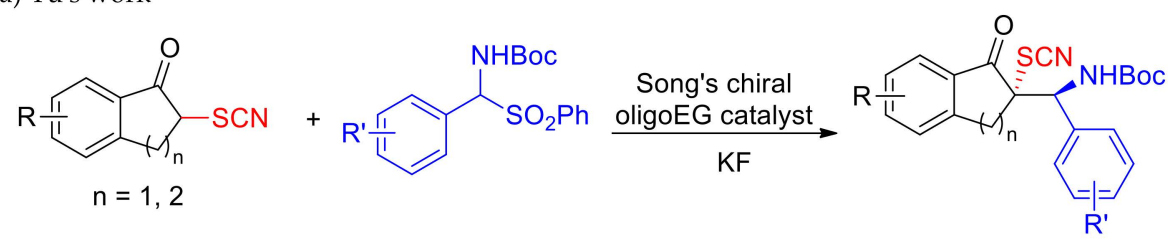

(b) This work<smiles>[R]C=C1Oc2ccc([R1])cc2C1=[N+]=[N-]</smiles><smiles>[R1]c1cc2c(cc1[R1])C(=O)C([Si]#N)C2</smiles>

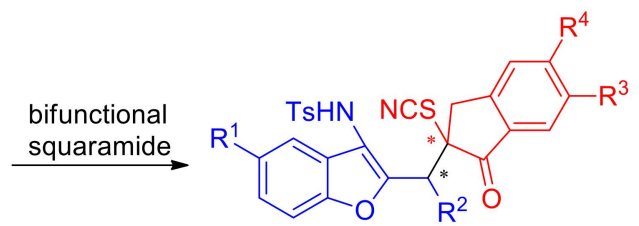

Scheme 1. The asymmetric Mannich reaction of $\alpha$-thiocyanoindanones and this work.

Therefore, in this paper, we envisioned a 1,4-Michael addition reaction between azadienes and $\alpha$-thiocyanoindanones, which could afford a series of optically active compounds 
containing benzofuran motif and thiocyano group with excellent diastereoselectivities and good enantioselectivities. This reaction not only provides a new strategy for the synthesis of thiocyanate compounds, but also the types of nucleophiles which react with azadienes are further expanded (Scheme 1b).

\section{Results and Discussion}

In the beginning, a series of organic catalysts were used to select the optimal catalyst for the 1,4-Michael addition reaction between azadienes and $\alpha$-thiocyanoindanones. We first used a quinine-derived squaramide $\mathbf{C} 1$ to catalyze 1,4-Michael addition reaction of azadiene $1 \mathrm{a}$ and $\alpha$-thiocyanoindanone $2 \mathrm{a}$ in dichloromethane solution. Fortunately, the target product 3aa was obtained with moderate yield and enantioselectivity (Table 1, entry 1). Encouraged by this result, several other catalysts C2-C9 (Figure 3) were screened to catalyze the 1,4-Michael addition reaction (Table 1, entries 2-9). However, no results better than $\mathbf{C} \mathbf{1}$ were obtained. Therefore, we still use $\mathbf{C} \mathbf{1}$ as the optimal catalyst.

Table 1. Establishment of optimal reaction conditions for azadiene and $\alpha$-thiocyanoindanone ${ }^{a}$.

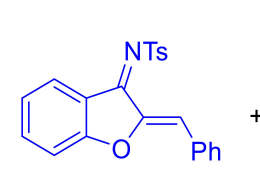

1a<smiles>N#CC1Cc2ccccc2C1=O</smiles>

2a

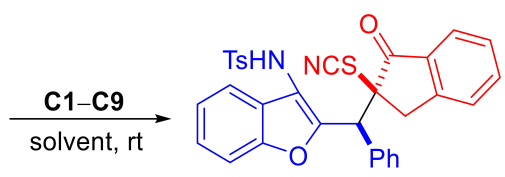

3aa

\begin{tabular}{|c|c|c|c|c|c|}
\hline Entry & Solvent & Catalyst & Yield $^{b}(\%)$ & $d r^{c}$ & $e r^{d}$ \\
\hline 1 & $\mathrm{CH}_{2} \mathrm{Cl}_{2}$ & C1 & 77 & $90: 10$ & $83: 17$ \\
\hline 2 & $\mathrm{CH}_{2} \mathrm{Cl}_{2}$ & $\mathrm{C} 2$ & 50 & $89: 11$ & $80: 20$ \\
\hline 3 & $\mathrm{CH}_{2} \mathrm{Cl}_{2}$ & C3 & 54 & $86: 14$ & $73: 27$ \\
\hline 4 & $\mathrm{CH}_{2} \mathrm{Cl}_{2}$ & C4 & 63 & $88: 12$ & $76: 24$ \\
\hline 5 & $\mathrm{CH}_{2} \mathrm{Cl}_{2}$ & C5 & 53 & $86: 14$ & $71: 29$ \\
\hline 6 & $\mathrm{CH}_{2} \mathrm{Cl}_{2}$ & C6 & 24 & $86: 14$ & $67: 33$ \\
\hline 7 & $\mathrm{CH}_{2} \mathrm{Cl}_{2}$ & C7 & 53 & $93: 7$ & $81: 19$ \\
\hline 8 & $\mathrm{CH}_{2} \mathrm{Cl}_{2}$ & C8 & 35 & $84: 16$ & $56: 44$ \\
\hline 9 & $\mathrm{CH}_{2} \mathrm{Cl}_{2}$ & C9 & 49 & $88: 12$ & $67: 33$ \\
\hline 10 & PhMe & C1 & 88 & $>95: 5$ & $81: 19$ \\
\hline 11 & THF & C1 & 56 & $>95: 5$ & $82: 18$ \\
\hline 12 & MTBE & C1 & 45 & $>95: 5$ & $78: 22$ \\
\hline 13 & EtOAc & C1 & 68 & $>95: 5$ & $86: 14$ \\
\hline 14 & 1,4-dioxane & C1 & 54 & $>95: 5$ & $86: 14$ \\
\hline 15 & $\mathrm{PhCF}_{3}$ & C1 & 82 & $>95: 5$ & $80: 20$ \\
\hline 16 & $\mathrm{CHCl}_{3}$ & C1 & 60 & $>95: 5$ & $79: 21$ \\
\hline 17 & $\mathrm{PhCl}$ & C1 & 67 & $>95: 5$ & $82: 18$ \\
\hline 18 & $\mathrm{CH}_{3} \mathrm{CN}$ & C1 & 28 & $>95: 5$ & $81: 19$ \\
\hline 19 & acetone & C1 & 46 & $>95: 5$ & $79: 21$ \\
\hline 20 & $\mathrm{ClCH}_{2} \mathrm{CH}_{2} \mathrm{Cl}$ & C1 & 83 & $76: 24$ & $81: 19$ \\
\hline 21 & $\mathrm{CCl}_{4}$ & C1 & 81 & $>95: 5$ & $70: 30$ \\
\hline 22 & dimethylbenzene & C1 & 36 & $76: 24$ & $63: 37$ \\
\hline 23 & $\mathrm{MeOH}$ & C1 & 24 & $>95: 5$ & $45: 55$ \\
\hline $24^{e}$ & EtOAc & C1 & 41 & $89: 11$ & $86: 14$ \\
\hline $25^{f}$ & EtOAc & C1 & 36 & $79: 21$ & $63: 37$ \\
\hline $26^{g}$ & EtOAc & C1 & 80 & $71: 29$ & $87: 13$ \\
\hline $27^{h}$ & EtOAc & C1 & 54 & $90: 10$ & $86: 14$ \\
\hline $28^{i}$ & EtOAc & C1 & 72 & $>95: 5$ & $90: 10$ \\
\hline $29^{j}$ & EtOAc & C1 & 86 & $93: 7$ & $85: 15$ \\
\hline $30^{k}$ & EtOAc & C1 & 49 & $92: 8$ & $84: 16$ \\
\hline
\end{tabular}

${ }^{a}$ Unless otherwise specified, reactions were carried out with 1 a $(0.05 \mathrm{mmol}), 2 \mathrm{a}(0.04 \mathrm{mmol})$ and catalyst $(5 \mathrm{~mol} \%)$ in solvent $(0.5 \mathrm{~mL})$ at room temperature. ${ }^{b}$ Isolated yield after column chromatography purification. ${ }^{c}$ Determined by ${ }^{1} \mathrm{H}$ NMR analysis. ${ }^{d}$ Enantiometric ratio was determined by HPLC analysis. ${ }^{e} 2.5 \mathrm{~mol} \%$ catalyst was used. $f^{1} 1 \mathrm{~mol} \%$ catalyst was used. $g 10$ mol\% catalyst was used. ${ }^{h}$ The reaction was performed at $0{ }^{\circ} \mathrm{C}$. ${ }^{i}$ The reaction was performed at $-10^{\circ} \mathrm{C}$. ${ }^{j}$ The reaction was performed at $-20^{\circ} \mathrm{C}$. ${ }^{k}$ The reaction was carried out with $1 \mathrm{a}(0.05 \mathrm{mmol}), 2 \mathrm{a}(0.04 \mathrm{mmol})$, and $\mathbf{C 1}(5 \mathrm{~mol} \%)$ in EtOAc $(2 \mathrm{~mL})$ at $-10{ }^{\circ} \mathrm{C}$. 


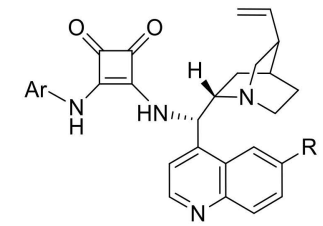

$\mathrm{C1}, \mathrm{Ar}=3,5-\left(\mathrm{CF}_{3}\right)_{2} \mathrm{C}_{6} \mathrm{H}_{3}, \mathrm{R}=\mathrm{OMe}$ $\mathrm{C} 2, \mathrm{Ar}=3,5-\left(\mathrm{CF}_{3}\right)_{2} \mathrm{C}_{6} \mathrm{H}_{3}, \mathrm{R}=\mathrm{H}$ $\mathrm{C} 3, \mathrm{Ar}=4-\mathrm{CF}_{3} \mathrm{C}_{6} \mathrm{H}_{4}, \mathrm{R}=\mathrm{OMe}$ $\mathrm{C} 4, \mathrm{Ar}=4-\mathrm{BBC}_{6} \mathrm{H}_{4}, \mathrm{R}=\mathrm{OMe}$

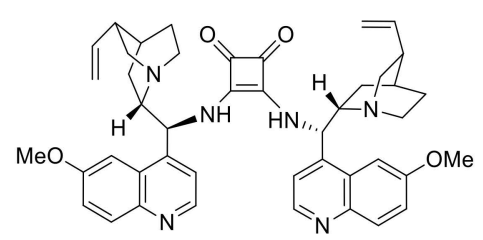

C7

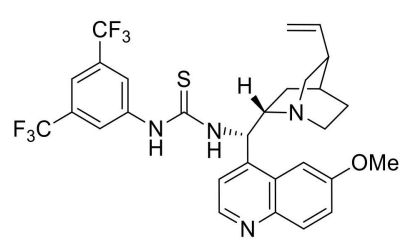

C5

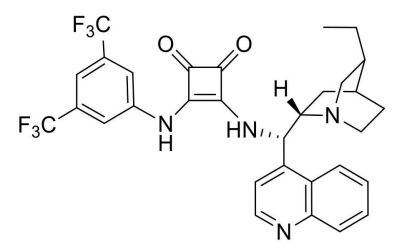

C8<smiles>O=c1c(Nc2cc(C(F)(F)F)cc(C(F)(F)F)c2)c(NC2CCCCC2N2CCCCC2)c1=O</smiles>

c6

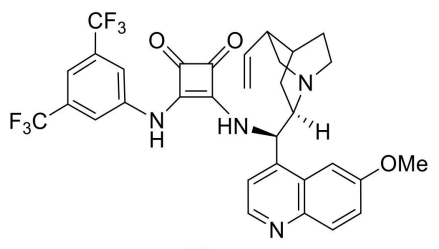

c9

Figure 3. The screened organocatalysts.

Next, the influence of the solvent effect on the reaction was examined. A total of 14 solvents (Table 1, entries 10-23) was evaluated. Surprisingly, when ethyl acetate was used, although the product yield was lower than that using dichloromethane, the enantioselectivity and stereoselectivity were better than using dichloromethane. Therefore, ethyl acetate was chosen as the best solvent. After the optimal solvent was determined, we believed that temperature and catalyst loading may also have influence on the reaction outcome. As expected, when the catalyst loading was reduced to $2.5 \mathrm{~mol} \%$, although the enantioselectivity was increased to 86:14 er, the diastereoselectivity decreased to 89:11 $d r$ (Table 1, entry 24). Considering that reducing the catalyst loading can increase the enantioselectivity, we decided to further reduce the catalyst loading to $1 \mathrm{~mol} \%$. Regrettably, both the $e r$ and $d r$ values decreased (Table 1 , entry 25). When the catalyst loading was increased to $10 \mathrm{~mol} \%$, the enantioselectivity increased to $87: 13 \mathrm{er}$, and the diastereoselectivity decreased to 71:29 $d r$. (Table 1, entry 26); therefore, we decided to still use $5 \mathrm{~mol} \%$ catalyst loading. We also tried to lower the temperature to $0,-10$, and $-20^{\circ} \mathrm{C}$ (Table 1 , entries 27-29). Fortunately, when the temperature was lowered to $-10^{\circ} \mathrm{C}$, the $\mathrm{er}$ value could be increased to 90:10 er. In addition, we reduced the concentration of the reaction solution and put azadiene and $\alpha$-thiocyanoindanone in $2 \mathrm{~mL}$ ethyl acetate. Compared with entry 28 , the results showed that the yield, enantioselectivity, and diastereoselectivity decreased (Table 1, entry 30). Therefore, the best result was obtained using $0.05 \mathrm{mmol} 1 \mathrm{a}, 0.04 \mathrm{mmol}$ 2a, and $5 \mathrm{~mol} \% \mathrm{C} 1$ in EtOAc at $-10^{\circ} \mathrm{C}$.

After the optimal reaction conditions were determined, the influence of different substituents on the substrates on the effect of this reaction was investigated. Firstly, the influence of different substituents in the azadienes 1 on the reaction was investigated, and the results are listed in Table 2 (entries 1-13). Regardless of whether $\mathrm{R}^{2}$ was an aromatic ring substituted with para-electron-withdrawing group or electron-donating group (Table 2, entries 2-7), the reaction of azadienes 1 with $\alpha$-thiocyanoindanone $2 a$ could basically maintain medium yields ( $58 \%$ to $87 \%$ ), moderate to excellent diastereoselectivity (75:25 $d r$ to $>95: 5 d r$ ), and good enantioselectivities (79:21 er to 90:10 er ). 
Table 2. Substrate scope of azadienes ${ }^{a}$.

\begin{tabular}{|c|c|c|c|c|c|}
\hline $\mathrm{R}^{1}$ & NTs & & $\frac{\text { nol } \% \text { C1 }}{\text { setate, }-10}$ & & \\
\hline Entry & $R^{1 / / R^{2}}(1)$ & 3 & Yield $^{b}(\%)$ & $d r^{c}$ & $e r^{d}$ \\
\hline 1 & $\mathrm{H} / \mathrm{Ph}(\mathbf{1 a})$ & $3 a a$ & 72 & $>95: 5$ & $90: 10$ \\
\hline 2 & $\mathrm{H} / p-\mathrm{BrC}_{6} \mathrm{H}_{4}(\mathbf{1} \mathbf{b})$ & $3 \mathbf{b a}$ & 68 & $80: 20$ & $89: 11$ \\
\hline 3 & $\mathrm{H} / p-\mathrm{MeC}_{6} \mathrm{H}_{4}(\mathbf{1 c})$ & $3 \mathrm{ca}$ & 87 & $>95: 5$ & $90: 10$ \\
\hline 4 & $\mathrm{H} / p-\mathrm{FC}_{6} \mathrm{H}_{4}(\mathbf{1 d})$ & $3 d a$ & 70 & $85: 15$ & $88: 12$ \\
\hline 5 & $\mathrm{H} / p-\mathrm{ClC}_{6} \mathrm{H}_{4}(\mathbf{1 e})$ & 3ea & 63 & $80: 20$ & $88: 12$ \\
\hline 6 & $\mathrm{H} / p-\mathrm{CF}_{3} \mathrm{C}_{6} \mathrm{H}_{4}(\mathbf{1 f})$ & $3 f a$ & 62 & $75: 25$ & $89: 11$ \\
\hline 7 & $\begin{array}{c}\mathrm{H} / p-\mathrm{MeOC}_{6} \mathrm{H}_{4} \\
(\mathbf{1 g})\end{array}$ & $3 g a$ & 58 & $>95: 5$ & 79:21 \\
\hline 8 & $\mathrm{H} / m-\mathrm{MeC}_{6} \mathrm{H}_{4}(\mathbf{1 h})$ & 3ha & 79 & $>95: 5$ & $90: 10$ \\
\hline 9 & $\mathrm{H} / m-\mathrm{BrC}_{6} \mathrm{H}_{4}(\mathbf{1 i})$ & $3 i a$ & 58 & $70: 30$ & $63: 37$ \\
\hline 10 & H/2-naphthyl (1j) & $3 \mathbf{j a}$ & 83 & $>95: 5$ & $90: 10$ \\
\hline 11 & $\mathrm{H} / 2$-thienyl (1k) & 3ka & 76 & $86: 14$ & $86: 14$ \\
\hline 12 & H/2-pyridinyl (11) & 3la & 78 & $>95: 5$ & $86: 14$ \\
\hline 13 & $\mathrm{Me} / \mathrm{Ph}(\mathbf{1 m})$ & $3 \mathrm{ma}$ & 60 & $>95: 5$ & $72: 28$ \\
\hline
\end{tabular}

${ }^{a}$ Unless otherwise noted, all reactions were carried out using $\mathbf{1}(0.05 \mathrm{mmol}), \mathbf{2 a}(0.04 \mathrm{mmol})$, and catalyst $\mathbf{C} \mathbf{1}(5 \mathrm{~mol} \%)$ in solvent $(0.5 \mathrm{~mL})$ at $-10{ }^{\circ} \mathrm{C} .{ }^{b}$ Isolated yield after column chromatography purification. ${ }^{c}$ The $d r$ values were determined by ${ }^{1} \mathrm{H}$ NMR analysis. ${ }^{d}$ Enantiometric ratio was determined by HPLC analysis.

When we used azadienes ( $\mathrm{R}^{2}$ is a meta-substituted aromatic ring) for the reaction (Table 2, entries 8 and 9), the enantioselectivity, yield, and diastereoselectivity of the electron-withdrawing group decreased. By contrast, the electron-donating group could maintain the enantioselectivity, yield, and diastereoselectivity. It is considered that the metabromo substitution makes the solubility of azadiene in ethyl acetate worse, which leads to a worsening of the chiral control effect. We also investigated the influence of the azadiene $\left(\mathrm{R}^{2}\right.$ is ortho-substituted aryl) on the reaction, which was affected by steric hindrance; it caused the reaction product to be only a trace amount. In addition, azadienes bearing 2-naphthyl, 2-thienyl, and 2-pyridinyl groups (Table 2, entries 10-12) also participated in the addition reaction to obtain corresponding products in good yields and er value. Finally, we also investigated azadiene when the $\mathrm{R}^{1}$ is methyl (Table 2, entries 13). Unfortunately, the enantioselectivity of this substrate has also decreased accordingly. Therefore, we found that the azadienes substituted by aryl groups at different positions have different control effects on the reaction. Generally speaking, the reaction has good universality for azadiene substrates.

In the next part, the effect of different substitutions of $\alpha$-thiocyanoindanone on the reaction was further investigated. We mainly examined the 6-electron withdrawing/donating group and 5-electron withdrawing/donating group-substituted $\alpha$-thiocyanoindanones. As shown in Table 3, entries 2-9, all the tried 5- and 6-substituted $\alpha$-thiocyanoindanones can undergo 1,4-Michael addition with azadiene 1a to give the target products in moderate yields ( $43 \%$ to $66 \%$ ). Among them, the electron-donating group substitution of $\alpha$-thiocyanoindanone is significantly better than the electron-withdrawing group substitution. The 6-position except-OMe-substituted $\alpha$-thiocyanindanone reacts with azadiene 1a to maintain excellent diastereoselectivity ( $>95: 5 d r)$. The product obtained by the reaction of $\alpha$-thiocyanoindanone substituted by the electron-donating group at the 6-position can maintain moderate yields and enantioselectivity (Table 3, entries 3 and 5). However, the enantioselectivities of the products decreased when the 6-fluoro- substituted and 6-bromosubstituted $\alpha$-thiocyanoindanones reacted with azadiene 1a (Table 3, entries 2 and 4). The diastereoselectivities and enantioselectivities of the products obtained by reacting of 5- 
substituted $\alpha$-thiocyanoindanones with azadiene 1a were all decreased (Table 3, entries 6-9). From this, we can see that different electron-withdrawing group substitutions of $\alpha$-thiocyanoindanone generally result in poor reaction effects.

Table 3. Substrate scope of $\alpha$-thiocyanoindanones ${ }^{a}$.

\begin{tabular}{|c|c|c|c|c|c|}
\hline & $\mathrm{Ph} \quad \mathrm{R}$ & & $\underset{\text { tate, }-10{ }^{\circ} \mathrm{C}}{\frac{1 \% \mathbf{C 1}}{\longrightarrow}}$ & $S$ & \\
\hline Entry & $R^{3} / R^{4}(2)$ & 3 & Yield $^{b}(\%)$ & $d r^{c}$ & $e r^{d}$ \\
\hline 1 & $\mathrm{H} / \mathrm{H}(\mathbf{2 a})$ & 3aa & 72 & $>95: 5$ & $90: 10$ \\
\hline 2 & $\mathrm{~F} / \mathrm{H}(\mathbf{2 b})$ & $3 a b$ & 61 & $>95: 5$ & $78: 22$ \\
\hline 3 & $\mathrm{Me} / \mathrm{H}(2 \mathrm{c})$ & $3 a c$ & 66 & $>95: 5$ & $87: 13$ \\
\hline 4 & $\mathrm{Br} / \mathrm{H}(\mathbf{2 d})$ & 3ad & 53 & $>95: 5$ & $70: 30$ \\
\hline 5 & $\mathrm{MeO} / \mathrm{H}(\mathbf{2 e})$ & $3 a e$ & 43 & $87: 13$ & $86: 14$ \\
\hline 6 & $\mathrm{H} / \mathrm{F}(\mathbf{2 f})$ & $3 a f$ & 45 & $86: 14$ & $74: 26$ \\
\hline 7 & $\mathrm{H} / \mathrm{Cl}(2 \mathrm{~g})$ & $3 a g$ & 61 & $84: 16$ & $62: 38$ \\
\hline 8 & $\mathrm{H} / \mathrm{Br}(\mathbf{2 h})$ & $3 \mathrm{ah}$ & 64 & $83: 17$ & $69: 31$ \\
\hline 9 & $\mathrm{H} / \mathrm{MeO}(\mathbf{2} \mathbf{i})$ & 3ai & 64 & $83: 17$ & $85: 15$ \\
\hline
\end{tabular}

${ }^{a}$ Unless otherwise noted, all reactions were carried out using $1(0.05 \mathrm{mmol}), \mathbf{2 a}(0.04 \mathrm{mmol})$, and catalyst $\mathrm{C} 1(5 \mathrm{~mol} \%)$ in ethyl acetate $(0.5 \mathrm{~mL})$ at $-10^{\circ} \mathrm{C} .{ }^{b}$ Isolated yield after column chromatography purification. ${ }^{c}$ The $d r$ values were determined by ${ }^{1} \mathrm{H}$ NMR analysis.

${ }^{d}$ Enantiometric ratio was determined by HPLC analysis.

The structures of the products obtained by the reaction of azadienes and $\alpha$-thiocyanoindanones were determined by NMR and HRMS. It is worth mentioning that because the enantioselectivity of $3 \mathbf{j a}$ is not very high (only 90:10 er) and cannot improve the enantioselectivity by recrystallization, we only obtained the relative configuration of the product through its single-crystal X-ray analysis (Figure 4) [30]. A similar phenomenon, only relative configuration, was provided owing to low enantioselectivity in Shi's report [31].

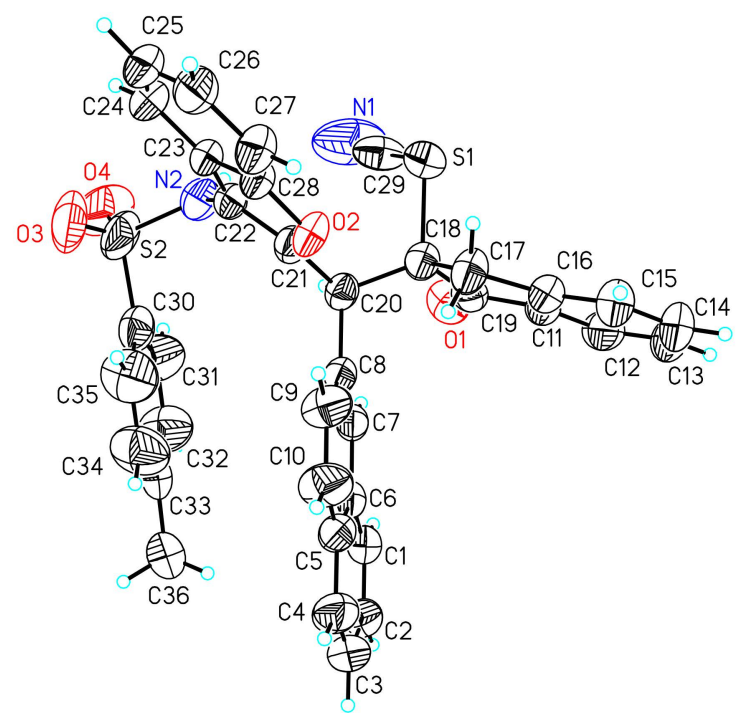

Figure 4. X-ray crystal structure of $3 \mathbf{j a}$.

Based on the experimental results and the relative configuration of $3 \mathbf{j a}$, we proposed a possible reaction pathway for the catalytic asymmetric Michael addition (Scheme 2). The 
2-thiocyano-1-indanone 2a is enolized and deprotonated via quinidine amine of chiral squaramide $\mathbf{C}$. Meanwhile, benzofuran-derived azadiene 1a is activated by catalyst $\mathbf{C 1}$ via forming hydrogen bonds. This dual activating mode of chiral squaramide $\mathbf{C} 1$ on the two substrates (transition state A) led to a stereoselective Michael addition to give an intermediate $\mathbf{B}$. Then, an intramolecular protonation of the intermediate $\mathbf{B}$ occurs to finish the Michael addition and afford product 3 aa with the observed relative configuration.

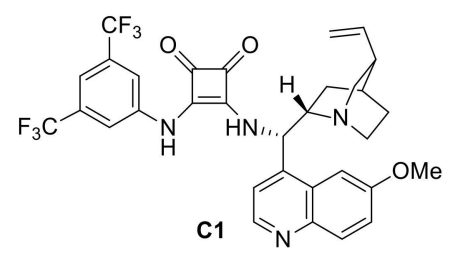

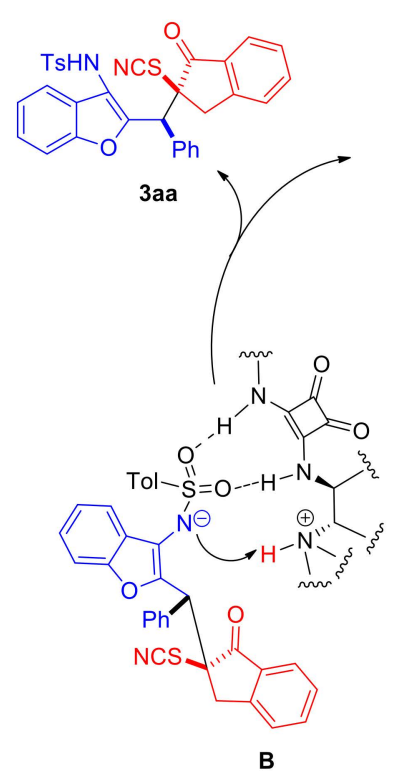<smiles>C#COC(=O)OC#C</smiles><smiles>N#CC1Cc2ccccc2C1=O</smiles>

Scheme 2. Proposed mechanism for the reaction.

\section{Conclusions}

All in all, we have successfully established the 1,4-Michael addition reaction of azadienes and $\alpha$-thiocyanoindanones catalyzed by a chiral bifunctional squaramide, and a series of optically active thiocyano compounds bearing both quaternary and tertiary double stereocenters were obtained. This reaction successfully constructs the thiocyano group into benzofuran derivatives under mild conditions, which provides a new strategy for the development and synthesis of chiral thiocyano compounds. Experimental details for the unsuccessful direct esterification of complex 7, the metathesis reactions and spectroscopic data can be found in the Supplementary Materials.

\section{Materials and Methods}

\subsection{General Information}

Commercially available compounds were used without further purification. Solvents were dried according to standard procedures. Column chromatography was performed with silica gel (200 300 mesh). Melting points were determined with an XT-4 meltingpoint apparatus and were uncorrected. ${ }^{1} \mathrm{H}$ NMR spectra were measured with Bruker Ascend $400 \mathrm{MHz}$ spectrometer; chemical shifts were reported in $\delta(\mathrm{ppm})$ units relative to tetramethylsilane (TMS) as internal standard. ${ }^{13} \mathrm{C}$ NMR spectra were measured at $100 \mathrm{MHz}$ with $400 \mathrm{MHz}$ spectrometer; chemical shifts were reported in ppm relative to tetramethylsilane and referenced to solvent peak $\left(\mathrm{CDCl}_{3}, \delta \mathrm{C}=77.00\right)$. High-resolution mass spectra (Electron spray ionization) were measured with an Agilent 6520 Accurate-Mass 
Q-TOF MS system equipped with an electrospray ionization (ESI) source. Optical rotations were measured with a Krüss P8000 polarimeter at the indicated concentration with the units of $\mathrm{g} / 100 \mathrm{~mL}$. Enantiomeric excesses were determined by chiral HPLC analysis using an Agilent 1200 LC instrument with a Daicel Chiralpak IA, IC, or ADH column. ${ }^{1} \mathrm{H}$ and ${ }^{13} \mathrm{C}$ NMR spectra for newly synthesized compounds, $\mathrm{X}$-ray single crystal data for product $3 \mathbf{j a}$ and copies of the HPLC chromatograms can be found in the Supplementary Materials.

\subsection{Materials}

$\mathbf{1 a}-\mathbf{1 m}$ were prepared according to the literature report [32,33]. 2a-2i were prepared according to the literature [29]. The chiral organocatalysts were prepared by following the reported procedures [34-37].

\subsection{Procedure for the Synthesis of Racemates of 3}

To a dried small bottle were added 1 (0.04 mmol), 2 (0.03 mmol), Et ${ }_{3} \mathrm{~N}$ (1.0 mg, $0.01 \mathrm{mmol}, 0.2 \mathrm{equiv})$, and EtOAc $(0.5 \mathrm{~mL})$. After stirring at room temperature for $12 \mathrm{~h}$, the reaction mixture was concentrated and directly purified by silica gel column chromatography to afford the racemates of 3 .

\subsection{Procedure for the Synthesis of Chiral Compounds 3}

To a dried small bottle were added $1(0.05 \mathrm{mmol}), 2(0.04 \mathrm{mmol})$, chiral organocatalyst C1 (1.3 mg, $0.002 \mathrm{mmol}$, and 0.05 equiv), and EtOAc $(0.5 \mathrm{~mL})$. The mixture was stirred at $-10{ }^{\circ} \mathrm{C}$; the reaction mixture was concentrated and directly purified by silica gel column chromatography to afford the desired products 3 .

4-methyl-N-(2-((1-oxo-2-thiocyanato-2,3-dihydro-1H-inden-2-yl)(phenyl)methyl)benzofuran-3-y l)benzenesulfonamide (3aa)

White solid; Flash column chromatography eluent (ethyl acetate/petroleum ether = 1:5); $16.0 \mathrm{mg}$ (72\% yield); m.p. 110-112 ${ }^{\circ} \mathrm{C}$. HPLC (Daicel Chiralpak IA, $n$-hexane/ethyl acetate $=80: 20$, flow rate $1.0 \mathrm{~mL} / \mathrm{min}$, detection at $254 \mathrm{~nm}): t_{R}=19.0 \mathrm{~min}($ minor $), t_{R}=22.7 \mathrm{~min}$ (major); 90:10 er. $[\alpha]_{\mathrm{D}}{ }^{25}=+69.1^{\circ}\left(c\right.$ 1.37, $\left.\mathrm{CH}_{2} \mathrm{Cl}_{2}\right) .{ }^{1} \mathrm{H}$ NMR $\left(400 \mathrm{MHz}, \mathrm{CDCl}_{3}\right): \delta 7.77$ $(\mathrm{d}, J=7.6 \mathrm{~Hz}, 1 \mathrm{H}, \mathrm{ArH}), 7.72(\mathrm{~d}, J=8.0 \mathrm{~Hz}, 1 \mathrm{H}, \mathrm{ArH}), 7.57-7.53(\mathrm{~m}, 2 \mathrm{H}, \mathrm{ArH}), 7.46(\mathrm{~d}$, $J=8.4 \mathrm{~Hz}, 2 \mathrm{H}, \mathrm{ArH}), 7.41-7.30(\mathrm{~m}, 4 \mathrm{H}, \mathrm{ArH}), 7.09-7.02(\mathrm{~m}, 5 \mathrm{H}, \mathrm{ArH}), 6.86(\mathrm{~d}, J=8.4 \mathrm{~Hz}$, 2H, ArH), 6.77 (s, 1H, NH), 5.05(s, 1H, CH), 4.01 (d, J = $\left.19.2 \mathrm{~Hz}, 1 \mathrm{H}, \mathrm{CH}_{2}\right), 3.23$ (d, $\left.J=18.8 \mathrm{~Hz}, 1 \mathrm{H}, \mathrm{CH}_{2}\right), 2.21\left(\mathrm{~s}, 3 \mathrm{H}, \mathrm{CH}_{3}\right) \mathrm{ppm} .{ }^{13} \mathrm{C} \mathrm{NMR}\left(100 \mathrm{MHz}, \mathrm{CDCl}_{3}\right): \delta 198.4,153.7$, $151.4,149.7,143.5,136.3,135.9,134.4,133.0,129.4,129.3,128.5,128.4,127.6,127.0,126.1$, $125.9,125.5,125.4,123.8,121.0,117.1,111.4,110.2,61.7,44.4,35.8,21.5$ ppm. HRMS (ESI): $m / z$ calcd. for $\mathrm{C}_{32} \mathrm{H}_{25} \mathrm{~N}_{2} \mathrm{O}_{4} \mathrm{~S}_{2}[\mathrm{M}+\mathrm{H}]^{+} 565.1250$, found 565.1263.

N-(2-((4-bromophenyl)(1-oxo-2-thiocyanato-2,3-dihydro-1H-inden-2-yl)methyl)benzofuran-3-yl)-4methylbenzenesulfonamide (3ba)

White solid; Flash column chromatography eluent (ethyl acetate/petroleum ether = 1:5); $17.5 \mathrm{mg}$ (68\% yield); m.p. 84-86 ${ }^{\circ} \mathrm{C}$. HPLC (Daicel Chiralpak IC, n-hexane/2-propanol = 80:20, flow rate $1.0 \mathrm{~mL} / \mathrm{min}$, detection at $254 \mathrm{~nm}$ ): $t_{R}=67.0 \mathrm{~min}$ (major), $t_{R}=78.9 \mathrm{~min}$ (minor); 89:11 er. $[\alpha]_{\mathrm{D}}{ }^{25}=+60^{\circ}\left(\mathrm{c} 0.32, \mathrm{CH}_{2} \mathrm{Cl}_{2}\right) .{ }^{1} \mathrm{H}$ NMR $(400 \mathrm{MHz}, \mathrm{CDCl} 3): \delta 7.78(\mathrm{~d}, J=8.0 \mathrm{~Hz}$, $1 \mathrm{H}, \mathrm{ArH}), 7.70(\mathrm{~d}, J=7.6 \mathrm{~Hz}, 1 \mathrm{H}, \mathrm{ArH}), 7.61-7.54(\mathrm{~m}, 2 \mathrm{H}, \mathrm{ArH}), 7.45(\mathrm{~d}, J=8.0 \mathrm{~Hz}, 2 \mathrm{H}$, ArH), 7.40-7.29 (m, 4H, ArH), $7.16(\mathrm{~d}, J=8.4 \mathrm{~Hz}, 2 \mathrm{H}, \mathrm{ArH}), 6.94-6.85$ (m, 5H, ArH + NH), 5.07 (s, 1H, CH), $3.93\left(\mathrm{~d}, \mathrm{~J}=18.8 \mathrm{~Hz}, 1 \mathrm{H}, \mathrm{CH}_{2}\right), 3.23\left(\mathrm{~d}, \mathrm{~J}=18.8 \mathrm{~Hz}, 1 \mathrm{H}, \mathrm{CH}_{2}\right), 2.26(\mathrm{~s}, 3 \mathrm{H}$, $\mathrm{CH}_{3}$ ) ppm. ${ }^{13} \mathrm{C}$ NMR $\left(100 \mathrm{MHz}, \mathrm{CDCl}_{3}\right): \delta 198.2,153.7,150.8,149.5,143.8,136.5,135.9$, 133.6, 132.8, 131.6, 131.0, 129.3, 128.7, 127.1, 126.2, 125.7, 125.62, 125.60, 124.0, 122.0, 121.1, $117.4,111.4,110.0,61.4,43.7,35.6,21.6 \mathrm{ppm}$. HRMS (ESI): $\mathrm{m} / z$ calcd. for $\mathrm{C}_{32} \mathrm{H}_{24}{ }^{79} \mathrm{BrN}_{2} \mathrm{O}_{4} \mathrm{~S}_{2}$ $[\mathrm{M}+\mathrm{H}]^{+}$643.0355, found 643.0348. calcd. for $\mathrm{C}_{32} \mathrm{H}_{24}{ }^{81} \mathrm{BrN}_{2} \mathrm{O}_{4} \mathrm{~S}_{2}[\mathrm{M}+\mathrm{H}]^{+}$645.0335, found 645.0332 .

4-methyl-N-(2-((1-oxo-2-thiocyanato-2,3-dihydro-1H-inden-2-yl)(p-tolyl)methyl)benzofuran-3-y l)benzenesulfonamide (3ca) 
White solid; Flash column chromatography eluent (ethyl acetate/petroleum ether = 1:5); $20.1 \mathrm{mg}$ (87\% yield); m.p. $112-114^{\circ} \mathrm{C}$. HPLC (Daicel Chiralpak IA, $n$-hexane/ethyl acetate $=85: 15$, flow rate $1.0 \mathrm{~mL} / \mathrm{min}$, detection at $254 \mathrm{~nm}): t_{R}=41.6 \mathrm{~min}($ minor $), t_{R}=45.4 \mathrm{~min}$ (major); 90:10 er. $[\alpha]_{\mathrm{D}}{ }^{25}=+84.7^{\circ}\left(\right.$ c $\left.0.78, \mathrm{CH}_{2} \mathrm{Cl}_{2}\right)$. ${ }^{1} \mathrm{H}$ NMR $\left(400 \mathrm{MHz}, \mathrm{CDCl}_{3}\right): \delta 7.77$ $(\mathrm{d}, J=7.6 \mathrm{~Hz}, 1 \mathrm{H}, \mathrm{ArH}), 7.71(\mathrm{~d}, J=7.2 \mathrm{~Hz}, 1 \mathrm{H}, \mathrm{ArH}), 7.58-7.54(\mathrm{~m}, 2 \mathrm{H}, \mathrm{ArH}), 7.47(\mathrm{~d}$, $J=8.0 \mathrm{~Hz}, 2 \mathrm{H}, \mathrm{ArH}), 7.40-7.29$ (m, 4H, ArH), 6.91-6.82 (m, 6H, ArH), 6.68 (s, 1H, NH), 5.00 $(\mathrm{s}, 1 \mathrm{H}, \mathrm{CH}), 4.00\left(\mathrm{~d}, J=18.8 \mathrm{~Hz}, 1 \mathrm{H}, \mathrm{CH}_{2}\right), 3.22\left(\mathrm{~d}, J=18.8 \mathrm{~Hz}, 1 \mathrm{H}, \mathrm{CH}_{2}\right), 2.21\left(\mathrm{~s}, 3 \mathrm{H}, \mathrm{CH}_{3}\right)$, $2.19\left(\mathrm{~s}, 3 \mathrm{H}, \mathrm{CH}_{3}\right)$ ppm. ${ }^{13} \mathrm{C} \mathrm{NMR}\left(100 \mathrm{MHz}, \mathrm{CDCl}_{3}\right): \delta 198.4,153.7,151.7,149.8,143.5$, $137.3,136.2,136.0,133.0,131.4,129.3,129.2,129.1,128.5,127.1,126.1,126.0,125.5,125.4$, $123.8,121.0,116.9,111.3,110.2,61.7,44.0,35.8,21.5,21.0$ ppm. HRMS (ESI): $m / z$ calcd. for $\mathrm{C}_{33} \mathrm{H}_{27} \mathrm{~N}_{2} \mathrm{O}_{4} \mathrm{~S}_{2}[\mathrm{M}+\mathrm{H}]^{+}$579.1407, found 579.1403.

N-(2-((4-fluorophenyl)(1-oxo-2-thiocyanato-2,3-dihydro-1H-inden-2-yl)methyl)benzofuran-3-yl)-4methylbenzenesulfonamide (3da)

White solid; Flash column chromatography eluent (ethyl acetate/petroleum ether = 1:5); $16.3 \mathrm{mg}\left(70 \%\right.$ yield); m.p. $108-110{ }^{\circ} \mathrm{C}$. HPLC (Daicel Chiralpak IC, $n$-hexane/2propanol $=70: 30$, flow rate $1.0 \mathrm{~mL} / \mathrm{min}$, detection at $254 \mathrm{~nm}$ ): $t_{R}=28.5 \mathrm{~min}$ (major), $t_{R}=35.2 \mathrm{~min}$ (minor); 88:12 er. $[\alpha]_{\mathrm{D}}{ }^{25}=+115.7^{\circ}\left(c 0.46, \mathrm{CH}_{2} \mathrm{Cl}_{2}\right) .{ }^{1} \mathrm{H}$ NMR $(400 \mathrm{MHz}$, $\left.\mathrm{CDCl}_{3}\right): \delta 7.78(\mathrm{~d}, J=7.6 \mathrm{~Hz}, 1 \mathrm{H}, \mathrm{ArH}), 7.68(\mathrm{~d}, J=8.0 \mathrm{~Hz}, 1 \mathrm{H}, \mathrm{ArH}), 7.60-7.54(\mathrm{~m}, 2 \mathrm{H}$, ArH), $7.47(\mathrm{~d}, J=8.4 \mathrm{~Hz}, 2 \mathrm{H}, \mathrm{ArH}), 7.41-7.29(\mathrm{~m}, 4 \mathrm{H}, \mathrm{ArH}), 7.06-7.03$ (m, 2H, ArH), 6.90 $(\mathrm{d}, J=8.0 \mathrm{~Hz}, 2 \mathrm{H}, \mathrm{ArH}), 6.81(\mathrm{~s}, 1 \mathrm{H}, \mathrm{NH}), 6.75-6.71(\mathrm{~m}, 2 \mathrm{H}, \mathrm{ArH}), 5.08(\mathrm{~s}, 1 \mathrm{H}, \mathrm{CH}), 3.98$ $\left(\mathrm{d}, J=18.8 \mathrm{~Hz}, 1 \mathrm{H}, \mathrm{CH}_{2}\right), 3.23\left(\mathrm{~d}, J=18.8 \mathrm{~Hz}, 1 \mathrm{H}, \mathrm{CH}_{2}\right), 2.24\left(\mathrm{~s}, 3 \mathrm{H}, \mathrm{CH}_{3}\right) \mathrm{ppm} .{ }^{13} \mathrm{C} \mathrm{NMR}$ $\left(100 \mathrm{MHz} \mathrm{CDCl}_{3}\right): \delta 198.4,161.9\left(\mathrm{~d},{ }^{1} J_{\mathrm{C}-\mathrm{F}}=246.4 \mathrm{~Hz}\right), 153.7,151.1,149.6,143.6,136.5$, $136.0,132.9,131.2\left(\mathrm{~d},{ }^{3} J_{\mathrm{C}-\mathrm{F}}=8.1 \mathrm{~Hz}\right), 130.29,130.26,129.4,128.7,127.1,126.1,125.7,125.6$ $\left(\mathrm{d},{ }^{4} J_{\mathrm{C}-\mathrm{F}}=1.9 \mathrm{~Hz}\right), 123.9,121.0,117.1,115.4\left(\mathrm{~d},{ }^{2} J_{\mathrm{C}-\mathrm{F}}=21.3 \mathrm{~Hz}\right), 111.4,110.1,61.5,43.6,35.6$, 21.4 ppm. HRMS (ESI): $m / z$ calcd. for $\mathrm{C}_{32} \mathrm{H}_{24} \mathrm{FN}_{2} \mathrm{O}_{4} \mathrm{~S}_{2}[\mathrm{M}+\mathrm{H}]^{+} 583.1156$, found 583.1143.

N-(2-((4-chlorophenyl)(1-oxo-2-thiocyanato-2,3-dihydro-1H-inden-2-yl)methyl)benzofuran-3-yl)-4methylbenzenesulfonamide (3ea)

White solid; Flash column chromatography eluent (ethyl acetate/petroleum ether = 1:5); $15.1 \mathrm{mg}$ (63\% yield), m.p. $114-116^{\circ} \mathrm{C}$. HPLC (Daicel Chiralpak IC, $n$-hexane/2propanol $=80: 20$, flow rate $1.0 \mathrm{~mL} / \mathrm{min}$, detection at $254 \mathrm{~nm}$ ): $t_{R}=65.4 \mathrm{~min}$ (major), $t_{R}=75.7$ min (minor); 88:12 er. $[\alpha]_{\mathrm{D}}{ }^{25}=+60.6^{\circ}\left(c 1.09, \mathrm{CH}_{2} \mathrm{Cl}_{2}\right) .{ }^{1} \mathrm{H}$ NMR $(400 \mathrm{MHz}$, $\left.\mathrm{CDCl}_{3}\right): \delta 7.78(\mathrm{~d}, J=7.6 \mathrm{~Hz}, 1 \mathrm{H}, \mathrm{ArH}), 7.70(\mathrm{~d}, J=7.6 \mathrm{~Hz}, 1 \mathrm{H}, \mathrm{ArH}), 7.61-7.54(\mathrm{~m}, 2 \mathrm{H}$, $\mathrm{ArH}), 7.45(\mathrm{~d}, J=8.4 \mathrm{~Hz}, 2 \mathrm{H}, \mathrm{ArH}), 7.42-7.37(\mathrm{~m}, 2 \mathrm{H}, \mathrm{ArH}), 7.35-7.30(\mathrm{~m}, 2 \mathrm{H}, \mathrm{ArH})$, 7.03-6.97 (m, 4H, ArH), $6.88(\mathrm{~d}, J=8.0 \mathrm{~Hz}, 2 \mathrm{H}, \mathrm{ArH}), 6.79(\mathrm{~s}, 1 \mathrm{H}, \mathrm{NH}), 5.07$ (s, 1H, CH), $3.94\left(\mathrm{~d}, J=19.2 \mathrm{~Hz}, 1 \mathrm{H}, \mathrm{CH}_{2}\right), 3.22\left(\mathrm{~d}, J=18.8 \mathrm{~Hz}, 1 \mathrm{H}, \mathrm{CH}_{2}\right), 2.25\left(\mathrm{~s}, 3 \mathrm{H}, \mathrm{CH}_{3}\right) \mathrm{ppm} .{ }^{13} \mathrm{C}$ NMR (100 MHz, $\left.\mathrm{CDCl}_{3}\right): \delta$ 198.2, 153.8, 151.0, 149.5, 143.8, 136.5, 136.0, 133.8, 133.0, 132.8, $130.8,129.3,128.8,128.6,127.1,126.2,125.8,125.7,125.6,124.0,121.1,117.3,111.4,110.0$, 61.4, 43.6, 35.6, 21.5 ppm. HRMS (ESI): $m / z$ calcd. for $\mathrm{C}_{32} \mathrm{H}_{24} \mathrm{ClN}_{2} \mathrm{O}_{4} \mathrm{~S}_{2}[\mathrm{M}+\mathrm{H}]^{+} 599.0861$, found 599.0859 .

4-methyl-N-(2-((1-oxo-2-thiocyanato-2,3-dihydro-1H-inden-2-yl)(4-(trifluoromethyl)phenyl)met hyl)benzofuran-3-yl)benzenesulfonamide (3fa)

White solid; Flash column chromatography eluent (ethyl acetate/petroleum ether = 1:5); $15.7 \mathrm{mg}\left(62 \%\right.$ yield); m.p. $113-115{ }^{\circ} \mathrm{C}$. HPLC (Daicel Chiralpak IC, $n$-hexane $/ 2-$ propanol $=85: 15$, flow rate $1.0 \mathrm{~mL} / \mathrm{min}$, detection at $254 \mathrm{~nm}$ ): $t_{R}=59.3 \mathrm{~min}$ (major), $t_{R}=70.6 \min$ (minor); 89:11 er. $[\alpha]_{\mathrm{D}}{ }^{25}=+52.2^{\circ}\left(\right.$ c $\left.0.48, \mathrm{CH}_{2} \mathrm{Cl}_{2}\right) .{ }^{1} \mathrm{H} \mathrm{NMR}\left(400 \mathrm{MHz}, \mathrm{CDCl}_{3}\right)$ : $\delta 7.79(\mathrm{~d}, J=7.6 \mathrm{~Hz}, 1 \mathrm{H}, \mathrm{ArH}), 7.64-7.55(\mathrm{~m}, 3 \mathrm{H}, \mathrm{ArH}), 7.47(\mathrm{~d}, J=8.4 \mathrm{~Hz}, 2 \mathrm{H}, \mathrm{ArH})$, $7.41-7.28(\mathrm{~m}, 6 \mathrm{H}, \mathrm{ArH}), 7.22(\mathrm{~d}, J=8.4 \mathrm{~Hz}, 2 \mathrm{H}, \mathrm{ArH}), 6.94-6.87(\mathrm{~m}, 3 \mathrm{H}, \mathrm{ArH}+\mathrm{NH}), 5.21$ (s, 1H, CH), $3.93\left(\mathrm{~d}, J=19.2 \mathrm{~Hz}, 1 \mathrm{H}, \mathrm{CH}_{2}\right), 3.27\left(\mathrm{~d}, J=18.8 \mathrm{~Hz}, 1 \mathrm{H}, \mathrm{CH}_{2}\right), 2.21\left(\mathrm{~s}, 3 \mathrm{H}, \mathrm{CH}_{3}\right)$ ppm. ${ }^{13} \mathrm{C} \mathrm{NMR}\left(100 \mathrm{MHz}, \mathrm{CDCl}_{3}\right): \delta 198.0,153.8,150.8,149.3,143.7,138.6,136.6,136.1$, $132.8,129.85\left(q,{ }^{2} J_{C-F}=32.3 \mathrm{~Hz}\right), 129.81,129.4,128.8,127.1,126.2,125.77,125.75,125.71$, $125.3\left(q,{ }^{3} J_{C-F}=3.7 \mathrm{~Hz}\right), 124.1,123.7\left(q,{ }^{1} J_{C-F}=270.5 \mathrm{~Hz}\right), 120.9,117.6,111.4,109.9,61.6$, 
44.1, 35.7, 21.3 ppm. HRMS (ESI): $m / z$ calcd. for $\mathrm{C}_{33} \mathrm{H}_{24} \mathrm{~F}_{3} \mathrm{~N}_{2} \mathrm{O}_{4} \mathrm{~S}_{2}[\mathrm{M}+\mathrm{H}]^{+}$633.1124, found 633.1131 .

4-methyl-N-(2-((1-oxo-2-thiocyanato-2,3-dihydro-1H-inden-2-yl)(4-(oxo-l6-methyl)phenyl)meth yl)benzofuran-3-yl)benzenesulfonamide (3ga)

White solid; Flash column chromatography eluent (ethyl acetate/petroleum ether $=1: 5$ ); $13.8 \mathrm{mg}$ ( $58 \%$ yield); m.p. $105-107^{\circ} \mathrm{C}$. HPLC (Daicel Chiralpak IC, $n$-hexane/ethyl acetate $=85: 15$, flow rate $1.0 \mathrm{~mL} / \mathrm{min}$, detection at $254 \mathrm{~nm}$ ): $t_{R}=68.1 \mathrm{~min}$ (major), $t_{R}=78.5 \mathrm{~min}$ (minor); 79:21 er. $[\alpha]_{\mathrm{D}}{ }^{25}=+27.1^{\circ}\left(c 0.51, \mathrm{CH}_{2} \mathrm{Cl}_{2}\right) .{ }^{1} \mathrm{H}$ NMR $\left(400 \mathrm{MHz}, \mathrm{CDCl}_{3}\right): \delta 7.77$ $(\mathrm{d}, J=7.6 \mathrm{~Hz}, 1 \mathrm{H}, \mathrm{ArH}), 7.71(\mathrm{~d}, J=7.2 \mathrm{~Hz}, 1 \mathrm{H}, \mathrm{ArH}), 7.58-7.53(\mathrm{~m}, 2 \mathrm{H}, \mathrm{ArH}), 7.48(\mathrm{~d}$, $J=8.4 \mathrm{~Hz}, 2 \mathrm{H}, \mathrm{ArH}), 7.40-7.29(\mathrm{~m}, 4 \mathrm{H}, \mathrm{ArH}), 6.96-6.94(\mathrm{~m}, 2 \mathrm{H}, \mathrm{ArH}), 6.90(\mathrm{~d}, J=8.0 \mathrm{~Hz}$, $2 \mathrm{H}, \mathrm{ArH}), 6.70(\mathrm{~s}, 1 \mathrm{H}, \mathrm{NH}), 6.58-6.54(\mathrm{~m}, 2 \mathrm{H}, \mathrm{ArH}), 5.00(\mathrm{~s}, 1 \mathrm{H}, \mathrm{CH}), 4.01(\mathrm{~d}, J=19.2 \mathrm{~Hz}$, $\left.1 \mathrm{H}, \mathrm{CH}_{2}\right), 3.68\left(\mathrm{~s}, 3 \mathrm{H}, \mathrm{CH}_{3}\right), 3.22\left(\mathrm{~d}, J=19.2 \mathrm{~Hz}, 1 \mathrm{H}, \mathrm{CH}_{2}\right), 2.22\left(\mathrm{~s}, 3 \mathrm{H}, \mathrm{CH}_{3}\right) \mathrm{ppm} .{ }^{13} \mathrm{C} \mathrm{NMR}$ $\left(100 \mathrm{MHz}, \mathrm{CDCl}_{3}\right): \delta 198.5,158.8,153.7,151.7,149.8,143.5,136.3,136.0,133.0,130.6,129.4$, $128.5,127.1,126.4,126.1,126.0,125.5,125.4,123.9,121.0,116.8,113.7,111.3,110.2,61.6,55.0$, 43.6, 35.8, 21.4 ppm. HRMS (ESI): $m / z$ calcd. for $\mathrm{C}_{33} \mathrm{H}_{26} \mathrm{~N}_{2} \mathrm{O}_{5} \mathrm{~S}_{2} \mathrm{Na}[\mathrm{M}+\mathrm{Na}]^{+} 617.1175$, found 617.1173 .

4-methyl-N-(2-((1-oxo-2-thiocyanato-2,3-dihydro-1H-inden-2-yl)(m-tolyl)methyl)benzofuran-3-y l)benzenesulfonamide (3ha)

White solid; Flash column chromatography eluent (ethyl acetate/ petroleum ether $=1: 5$ ); $18.3 \mathrm{mg}$ (79\% yield); m.p. $102-104{ }^{\circ} \mathrm{C}$. HPLC (Daicel Chiralpak IA, $n$-hexane/ethyl acetate $=80: 20$, flow rate $1.0 \mathrm{~mL} / \mathrm{min}$, detection at $254 \mathrm{~nm}$ ): $t_{R}=18.2 \mathrm{~min}$ (minor), $t_{R}=20.2 \mathrm{~min}$ (major); 90:10 er. $[\alpha]_{\mathrm{D}}{ }^{25}=+64.6^{\circ}\left(c 0.50, \mathrm{CH}_{2} \mathrm{Cl}_{2}\right) .{ }^{1} \mathrm{H}$ NMR $\left(400 \mathrm{MHz}, \mathrm{CDCl}_{3}\right): \delta 7.78$ $(\mathrm{d}, J=7.6 \mathrm{~Hz}, 1 \mathrm{H}, \mathrm{ArH}), 7.74(\mathrm{~d}, J=7.6 \mathrm{~Hz}, 1 \mathrm{H}, \mathrm{ArH}), 7.57-7.54(\mathrm{~m}, 2 \mathrm{H}, \mathrm{ArH}), 7.47(\mathrm{~d}$, $J=8.4 \mathrm{~Hz}, 1 \mathrm{H}, \mathrm{ArH}), 7.41-7.31(\mathrm{~m}, 4 \mathrm{H}, \mathrm{ArH}), 6.93-6.82(\mathrm{~m}, 6 \mathrm{H}, \mathrm{ArH}), 6.72(\mathrm{~s}, 1 \mathrm{H}, \mathrm{NH})$, $5.02(\mathrm{~s}, 1 \mathrm{H}, \mathrm{CH}), 4.00\left(\mathrm{~d}, J=18.8 \mathrm{~Hz}, 1 \mathrm{H}, \mathrm{CH}_{2}\right), 3.23\left(\mathrm{~d}, J=19.2 \mathrm{~Hz}, 1 \mathrm{H}, \mathrm{CH}_{2}\right), 2.20(\mathrm{~s}, 3 \mathrm{H}$, $\left.\mathrm{CH}_{3}\right), 2.15\left(\mathrm{~s}, 3 \mathrm{H}, \mathrm{CH}_{3}\right) \mathrm{ppm} .{ }^{13} \mathrm{C} \mathrm{NMR}\left(100 \mathrm{MHz}, \mathrm{CDCl}_{3}\right): \delta 198.4,153.7,151.5,149.8,143.4$, 138.0, 136.2, 136.0, 134.3, 133.1, 129.9, 129.3, 128.5, 128.4, 128.3, 127.1, 126.4, 126.1, 126.0,

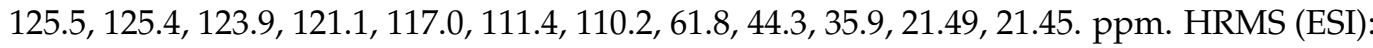
$m / z$ calcd. for $\mathrm{C}_{33} \mathrm{H}_{27} \mathrm{~N}_{2} \mathrm{O}_{4} \mathrm{~S}_{2}[\mathrm{M}+\mathrm{H}]^{+} 579.1407$, found 579.1419.

$\mathrm{N}$-(2-((3-bromophenyl)(1-oxo-2-thiocyanato-2,3-dihydro-1H-inden-2-yl)methyl)benzofuran-3-yl)-4methylbenzenesulfonamide (3ia)

White solid; Flash column chromatography eluent (ethyl acetate/petroleum ether $=1: 5$ ); $14.9 \mathrm{mg}$ ( $58 \%$ yield); m.p. $111-113^{\circ} \mathrm{C}$. HPLC (Daicel Chiralpak IA, $n$-hexane/ethyl acetate $=85: 15$, flow rate $1.0 \mathrm{~mL} / \mathrm{min}$, detection at $254 \mathrm{~nm}$ ): $t_{\mathrm{R}}=35.0 \mathrm{~min}$ (minor), $t_{\mathrm{R}}=38.9 \mathrm{~min}$ (major); 63:37 er. $[\alpha]_{\mathrm{D}}{ }^{25}=+51.0^{\circ}\left(c 0.82, \mathrm{CH}_{2} \mathrm{Cl}_{2}\right) .{ }^{1} \mathrm{H}$ NMR $\left(400 \mathrm{MHz}, \mathrm{CDCl}_{3}\right): \delta 7.80$ $(\mathrm{d}, J=7.6 \mathrm{~Hz}, 1 \mathrm{H}, \mathrm{ArH}), 7.73(\mathrm{~d}, J=7.6 \mathrm{~Hz}, 1 \mathrm{H}, \mathrm{ArH}), 7.61-7.56(\mathrm{~m}, 2 \mathrm{H}, \mathrm{ArH}), 7.46(\mathrm{~d}$, $J=8.4 \mathrm{~Hz}, 2 \mathrm{H}, \mathrm{ArH}), 7.43-7.31(\mathrm{~m}, 4 \mathrm{H}, \mathrm{ArH}), 7.23-7.18(\mathrm{~m}, 2 \mathrm{H}, \mathrm{ArH}), 7.01(\mathrm{~d}, J=8.0 \mathrm{~Hz}$, $1 \mathrm{H}, \mathrm{ArH}), 6.94-6.90(\mathrm{~m}, 1 \mathrm{H}, \mathrm{ArH}), 6.78(\mathrm{~s}, 1 \mathrm{H}, \mathrm{NH}), 5.01(\mathrm{~s}, 1 \mathrm{H}, \mathrm{CH}), 3.94(\mathrm{~d}, J=18.8 \mathrm{~Hz}$, $\left.1 \mathrm{H}, \mathrm{CH}_{2}\right), 3.23\left(\mathrm{~d}, J=19.2 \mathrm{~Hz}, 1 \mathrm{H}, \mathrm{CH}_{2}\right), 2.23\left(\mathrm{~s}, 3 \mathrm{H}, \mathrm{CH}_{3}\right) \mathrm{ppm} .{ }^{13} \mathrm{C} \mathrm{NMR}(100 \mathrm{MHz}$, $\left.\mathrm{CDCl}_{3}\right): \delta 198.1,153.8,150.5,149.5,143.6,136.7,136.5,135.8,132.9,132.1,130.9,129.9,129.4$, 128.7, 128.1, 127.1, 126.2, 125.68, 125.66, 124.0, 122.5, 121.1, 117.6, 111.5, 109.9, 61.6, 43.9, 35.7, 21.6 ppm. HRMS (ESI): $m / z$ calcd. for $\mathrm{C}_{32} \mathrm{H}_{24}{ }^{79} \mathrm{BrN}_{2} \mathrm{O}_{4} \mathrm{~S}_{2}[\mathrm{M}+\mathrm{H}]^{+}$643.0355, found 643.0354. calcd. for $\mathrm{C}_{32} \mathrm{H}_{24}{ }^{81} \mathrm{BrN}_{2} \mathrm{O}_{4} \mathrm{~S}_{2}[\mathrm{M}+\mathrm{H}]^{+}$645.0335, found 64.0334 .

4-methyl-N-(2-(naphthalen-1-yl(1-oxo-2-thiocyanato-2,3-dihydro-1H-inden-2-yl)methyl)benzofuran3-yl)benzenesulfonamide (3ja)

White solid; Flash column chromatography eluent (ethyl acetate/petroleum ether $=1: 5$ ); $20.4 \mathrm{mg}$ ( $83 \%$ yield); m.p. $118-120^{\circ} \mathrm{C}$. HPLC (Daicel Chiralpak IC, $n$-hexane/ethyl acetate $=85: 15$, flow rate $1.0 \mathrm{~mL} / \mathrm{min}$, detection at $254 \mathrm{~nm}$ ): $t_{\mathrm{R}}=52.7 \mathrm{~min}$ (major), $t_{\mathrm{R}}=60.9 \mathrm{~min}$ (minor); $90: 10$ er. $[\alpha]_{\mathrm{D}}{ }^{25}=+65.4^{\circ}\left(c 0.41, \mathrm{CH}_{2} \mathrm{Cl}_{2}\right) .{ }^{1} \mathrm{H}$ NMR $\left(400 \mathrm{MHz}, \mathrm{CDCl}_{3}\right): \delta 7.85(\mathrm{~d}, J=7.6 \mathrm{~Hz}$, $1 \mathrm{H}, \mathrm{ArH}), 7.77(\mathrm{~d}, J=7.6 \mathrm{~Hz}, 1 \mathrm{H}, \mathrm{ArH}), 7.70-7.61(\mathrm{~m}, 3 \mathrm{H}, \mathrm{ArH}), 7.52-7.29(\mathrm{~m}, 10 \mathrm{H}, \mathrm{ArH})$, $7.25(\mathrm{~d}, J=7.2 \mathrm{~Hz}, 1 \mathrm{H}, \mathrm{ArH}), 7.15(\mathrm{~d}, J=8.4 \mathrm{~Hz}, 1 \mathrm{H}, \mathrm{ArH}), 6.87(\mathrm{~s}, 1 \mathrm{H}, \mathrm{NH}), 6.53(\mathrm{~d}$, 
$J=8.0 \mathrm{~Hz}, 2 \mathrm{H}, \mathrm{ArH}), 5.25(\mathrm{~s}, 1 \mathrm{H}, \mathrm{CH}), 4.00\left(\mathrm{~d}, J=18.8 \mathrm{~Hz}, 1 \mathrm{H}, \mathrm{CH}_{2}\right), 3.24(\mathrm{~d}, J=19.2 \mathrm{~Hz}$, $\left.1 \mathrm{H}, \mathrm{CH}_{2}\right), 1.68\left(\mathrm{~s}, 3 \mathrm{H}, \mathrm{CH}_{3}\right)$ ppm. ${ }^{13} \mathrm{C}$ NMR $\left(100 \mathrm{MHz}, \mathrm{CDCl}_{3}\right): \delta$ 198.3, 153.9, 151.2, $149.5,143.5,136.3,135.6,132.9,132.8,132.3,132.0,129.1,129.0,128.6,128.2,128.1,127.4$, $126.9,126.54,126.51,126.4,126.1,126.0,125.6,125.5,123.9,121.4,117.3,111.4,110.3,61.7$, 44.1, 35.7, 20.8 ppm. HRMS (ESI): $m / z$ calcd. for $\mathrm{C}_{36} \mathrm{H}_{26} \mathrm{~N}_{2} \mathrm{O}_{4} \mathrm{~S}_{2} \mathrm{Na}[\mathrm{M}+\mathrm{Na}]^{+} 637.1226$, found 637.1228 .

4-methyl-N-(2-((1-oxo-2-thiocyanato-2,3-dihydro-1H-inden-2-yl)(thiophen-2-yl)methyl)benzofuran3-yl)benzenesulfonamide (3ka)

White solid; Flash column chromatography eluent (ethyl acetate/petroleum ether $=1: 5$ ); $17.6 \mathrm{mg}$ (76\% yield); m.p. $110-112^{\circ} \mathrm{C}$. HPLC (Daicel Chiralpak IC, $n$-hexane/ethyl acetate $=85: 15$, flow rate $1.0 \mathrm{~mL} / \mathrm{min}$, detection at $254 \mathrm{~nm}$ ): $t_{\mathrm{R}}=58.1 \mathrm{~min}$ (major), $t_{\mathrm{R}}=75.1 \mathrm{~min}$ (minor); 86:14 er. $[\alpha]_{\mathrm{D}}{ }^{25}=+61.5^{\circ}\left(\right.$ c 1.03, $\left.\mathrm{CH}_{2} \mathrm{Cl}_{2}\right) .{ }^{1} \mathrm{H}$ NMR $\left(400 \mathrm{MHz}, \mathrm{CDCl}_{3}\right): \delta 7.83(\mathrm{~d}$, $J=7.6 \mathrm{~Hz}, 1 \mathrm{H}, \mathrm{ArH}), 7.74(\mathrm{~d}, J=8.0 \mathrm{~Hz}, 1 \mathrm{H}, \mathrm{ArH}), 7.60(\mathrm{t}, J=7.6 \mathrm{~Hz}, 1 \mathrm{H}, \mathrm{ArH}), 7.56-7.53$ $(\mathrm{m}, 3 \mathrm{H}, \mathrm{ArH}), 7.43-7.31(\mathrm{~m}, 4 \mathrm{H}, \mathrm{ArH}), 7.00\left(\mathrm{dd}, J_{1}=5.0 \mathrm{~Hz}, J_{2}=0.8 \mathrm{~Hz}, 1 \mathrm{H}, \mathrm{ArH}\right), 6.95$ $(\mathrm{d}, J=8.0 \mathrm{~Hz}, 2 \mathrm{H}, \mathrm{ArH}), 6.80(\mathrm{~s}, 1 \mathrm{H}, \mathrm{NH}), 6.68-6.66(\mathrm{~m}, 1 \mathrm{H}, \mathrm{ArH}), 6.47(\mathrm{~d}, J=3.6 \mathrm{~Hz}, 1 \mathrm{H}$, $\mathrm{ArH}), 5.22(\mathrm{~s}, 1 \mathrm{H}, \mathrm{CH}), 4.05\left(\mathrm{~d}, J=18.8 \mathrm{~Hz}, 1 \mathrm{H}, \mathrm{CH}_{2}\right), 3.16\left(\mathrm{~d}, J=19.2 \mathrm{~Hz}, 1 \mathrm{H}, \mathrm{CH}_{2}\right), 2.24(\mathrm{~s}$, $\left.3 \mathrm{H}, \mathrm{CH}_{3}\right)$ ppm. ${ }^{13} \mathrm{C}$ NMR $\left(100 \mathrm{MHz}, \mathrm{CDCl}_{3}\right): \delta 198.1,153.7,150.3,150.0,143.6,136.3,136.0$, 135.9, 133.0, 129.4, 128.7, 128.6, 127.1, 126.5, 126.3, 125.9, 125.8, 125.7, 125.6, 124.0, 121.0, 117.1, 111.5, 110.1, 77.3, 77.0, 76.7, 61.2, 40.5, 35.9, $21.6 \mathrm{ppm}$. HRMS (ESI): $\mathrm{m} / \mathrm{z}$ calcd. for $\mathrm{C}_{30} \mathrm{H}_{23} \mathrm{~N}_{2} \mathrm{O}_{4} \mathrm{~S}_{3}[\mathrm{M}+\mathrm{H}]^{+}$571.0814, found 571.0818.

4-methyl-N-(2-((1-oxo-2-thiocyanato-2,3-dihydro-1H-inden-2-yl)(pyridin-3-yl)methyl)benzofuran3-yl)benzenesulfonamide (31a)

White solid; Flash column chromatography eluent (ethyl acetate/petroleum ether $=1: 2$ ); $17.6 \mathrm{mg}$ (78\% yield); m.p. $104-106{ }^{\circ} \mathrm{C}$. HPLC (Daicel Chiralpak ADH, $n$-hexane/2-propanol $=85: 15$, flow rate $1.0 \mathrm{~mL} / \mathrm{min}$, detection at $254 \mathrm{~nm}$ ): $t_{\mathrm{R}}=76.7 \mathrm{~min}$ (major), $t_{\mathrm{R}}=106.2 \mathrm{~min}$ (minor); 86:14 er. $[\alpha]_{\mathrm{D}}{ }^{25}=+35.3^{\circ}\left(\mathrm{c} 0.68, \mathrm{CH}_{2} \mathrm{Cl}_{2}\right) .{ }^{1} \mathrm{H}$ NMR $\left(400 \mathrm{MHz}, \mathrm{CDCl}_{3}\right): \delta 8.43-8.37$ $(\mathrm{m}, 2 \mathrm{H}, \mathrm{ArH}), 7.79(\mathrm{~d}, J=8.0 \mathrm{~Hz}, 1 \mathrm{H}, \mathrm{ArH}), 7.60-7.53(\mathrm{~m}, 3 \mathrm{H}, \mathrm{ArH}), 7.46(\mathrm{~d}, J=8.4 \mathrm{~Hz}$, $3 \mathrm{H}, \mathrm{ArH}), 7.40-7.34(\mathrm{~m}, 3 \mathrm{H}, \mathrm{ArH}), 7.25(\mathrm{t}, J=7.6 \mathrm{~Hz}, 1 \mathrm{H}, \mathrm{ArH}), 7.10-7.07(\mathrm{~m}, 1 \mathrm{H}, \mathrm{ArH})$, 7.00-6.94 (m, 3H, ArH + NH), $5.20(\mathrm{~s}, 1 \mathrm{H}, \mathrm{CH}), 4.00\left(\mathrm{~d}, J=19.2 \mathrm{~Hz}, 1 \mathrm{H}, \mathrm{CH}_{2}\right), 3.29(\mathrm{~d}$, $\left.J=19.2 \mathrm{~Hz}, 1 \mathrm{H}, \mathrm{CH}_{2}\right), 2.26\left(\mathrm{~s}, 3 \mathrm{H}, \mathrm{CH}_{3}\right) \mathrm{ppm} .{ }^{13} \mathrm{C} \mathrm{NMR}\left(100 \mathrm{MHz}, \mathrm{CDCl}_{3}\right): \delta 197.9,153.8$, $150.3,149.3,148.1,143.9,138.2,136.7,136.0,132.8,131.13,132.11,129.5,128.9,127.2,126.2$, $125.8,125.7,125.4,124.0,123.5,120.5,117.8,111.5,109.6,61.6,42.8,35.9,21.5$ ppm. HRMS (ESI): $m / z$ calcd. for $\mathrm{C}_{31} \mathrm{H}_{24} \mathrm{~N}_{3} \mathrm{O}_{4} \mathrm{~S}_{2}[\mathrm{M}+\mathrm{H}]^{+}$566.1203, found 566.1207.

4-methyl-N-(5-methyl-2-((1-oxo-2-thiocyanato-2,3-dihydro-1H-inden-2-yl)(phenyl)methyl)benzofuran3-yl)benzenesulfonamide (3ma)

White solid; Flash column chromatography eluent (ethyl acetate/petroleum ether $=1: 5$ ); $13.9 \mathrm{mg}\left(60 \%\right.$ yield); m.p. $116-118{ }^{\circ} \mathrm{C}$. HPLC (Daicel Chiralpak IC, $n$-hexane/ethyl acetate $=80: 20$, flow rate $1.0 \mathrm{~mL} / \mathrm{min}$, detection at $254 \mathrm{~nm}$ ): $t_{R}=20.9 \mathrm{~min}$ (minor), $t_{R}=24.9 \mathrm{~min}$ (major); $72: 28$ er. $[\alpha]_{\mathrm{D}}{ }^{25}=+51.8^{\circ}\left(\right.$ c $\left.0.84, \mathrm{CH}_{2} \mathrm{Cl}_{2}\right) .{ }^{1} \mathrm{H}$ NMR $\left(400 \mathrm{MHz}, \mathrm{CDCl}_{3}\right): \delta 7.76(\mathrm{~d}$, $J=7.6 \mathrm{~Hz}, 1 \mathrm{H}, \mathrm{ArH}), 7.54\left(\mathrm{td}, J_{1}=7.6 \mathrm{~Hz}, J_{2}=0.8 \mathrm{~Hz}, 1 \mathrm{H}, \mathrm{ArH}\right), 7.48(\mathrm{~d}, J=8.4 \mathrm{~Hz}, 2 \mathrm{H}$, ArH), 7.44-7.42 (m, 2H, ArH), $7.35(\mathrm{t}, J=7.4 \mathrm{~Hz}, 1 \mathrm{H}, \mathrm{ArH}), 7.30(\mathrm{~d}, J=8.0 \mathrm{~Hz}, 1 \mathrm{H}, \mathrm{ArH})$, $7.19\left(\mathrm{dd}, J_{1}=8.6 \mathrm{~Hz}, J_{2}=1.4 \mathrm{~Hz}, 1 \mathrm{H}, \mathrm{ArH}\right), 7.09-7.00(\mathrm{~m}, 5 \mathrm{H}, \mathrm{ArH}), 6.87(\mathrm{~d}, J=8.0 \mathrm{~Hz}, 2 \mathrm{H}$, $\mathrm{ArH}), 6.64(\mathrm{~s}, 1 \mathrm{H}, \mathrm{NH}), 5.01(\mathrm{~s}, 1 \mathrm{H}, \mathrm{CH}), 3.99\left(\mathrm{~d}, J=19.2 \mathrm{~Hz}, 1 \mathrm{H}, \mathrm{CH}_{2}\right), 3.21(\mathrm{~d}, J=19.2 \mathrm{~Hz}$, $\left.1 \mathrm{H}, \mathrm{CH}_{2}\right), 2.44\left(\mathrm{~s}, 3 \mathrm{H}, \mathrm{CH}_{3}\right), 2.22\left(\mathrm{~s}, 3 \mathrm{H}, \mathrm{CH}_{3}\right) \mathrm{ppm} .{ }^{13} \mathrm{C} \mathrm{NMR}\left(100 \mathrm{MHz}, \mathrm{CDCl}_{3}\right): \delta 198.4$, 152.2, 151.7, 149.7, 143.4, 136.2, 136.1, 134.5, 133.6, 133.0, 129.4, 129.3, 128.5, 128.4, 127.6, 127.1, 126.8, 126.1, 126.0, 125.5, 120.5, 116.8, 110.9, 110.2, 61.8, 44.4, 35.8, 21.5, $21.3 \mathrm{ppm}$. HRMS (ESI): $m / z$ calcd. for $\mathrm{C}_{33} \mathrm{H}_{27} \mathrm{~N}_{2} \mathrm{O}_{4} \mathrm{~S}_{2}[\mathrm{M}+\mathrm{H}]^{+} 579.1407$, found 579.1394.

N-(2-((6-fluoro-1-oxo-2-thiocyanato-2,3-dihydro-1H-inden-2-yl)(phenyl)methyl)benzofuran-3-yl)4-methylbenzenesulfonamide (3ab)

White solid; Flash column chromatography eluent (ethyl acetate/petroleum ether $=1: 5$ ); $14.2 \mathrm{mg}$ (61\% yield); m.p. $94-96^{\circ} \mathrm{C}$. HPLC (Daicel Chiralpak IC, $n$-hexane/2-propanol $=80: 20$, 
flow rate $1.0 \mathrm{~mL} / \mathrm{min}$, detection at $254 \mathrm{~nm}$ ): $t_{\mathrm{R}}=63.2 \mathrm{~min}$ (minor), $t_{\mathrm{R}}=80.0$ min (major); $78: 22$ er. $[\alpha]_{\mathrm{D}}{ }^{25}=+24.9^{\circ}\left(c 0.36, \mathrm{CH}_{2} \mathrm{Cl}_{2}\right) .{ }^{1} \mathrm{H}$ NMR $\left(400 \mathrm{MHz}, \mathrm{CDCl}_{3}\right): \delta 7.68(\mathrm{~d}, J=7.6 \mathrm{~Hz}$, 1H, ArH), $7.55(\mathrm{~d}, J=8.0 \mathrm{~Hz}, 1 \mathrm{H}, \mathrm{ArH}), 7.46(\mathrm{~d}, J=8.0 \mathrm{~Hz}, 2 \mathrm{H}, \mathrm{ArH}), 7.41-7.37$ (m, 2H, $\mathrm{ArH}), 7.33-7.24(\mathrm{~m}, 3 \mathrm{H}, \mathrm{ArH}), 7.12-7.01(\mathrm{~m}, 5 \mathrm{H}, \mathrm{ArH}), 6.88(\mathrm{~d}, J=8.0 \mathrm{~Hz}, 2 \mathrm{H}, \mathrm{ArH})$, $6.71(\mathrm{~s}, 1 \mathrm{H}, \mathrm{NH}), 5.04(\mathrm{~s}, 1 \mathrm{H}, \mathrm{CH}), 4.00\left(\mathrm{~d}, J=18.8 \mathrm{~Hz}, 1 \mathrm{H}, \mathrm{CH}_{2}\right), 3.21(\mathrm{~d}, J=18.8 \mathrm{~Hz}, 1 \mathrm{H}$, $\left.\mathrm{CH}_{2}\right), 2.22\left(\mathrm{~s}, 3 \mathrm{H}, \mathrm{CH}_{3}\right)$ ppm. ${ }^{13} \mathrm{C} \mathrm{NMR}\left(100 \mathrm{MHz}, \mathrm{CDCl}_{3}\right): \delta 197.7\left(\mathrm{~d},{ }^{4} J_{\mathrm{C}-\mathrm{F}}=3.0 \mathrm{~Hz}\right)$, $162.6\left(\mathrm{~d},{ }^{1} J_{\mathrm{C}-\mathrm{F}}=249.0 \mathrm{~Hz}\right), 153.7,151.2,145.2\left(\mathrm{~d},{ }^{4} J_{\mathrm{C}-\mathrm{F}}=2.0 \mathrm{~Hz}\right), 143.5,135.9,134.8(\mathrm{~d}$, $\left.{ }^{3} J_{\mathrm{C}-\mathrm{F}}=7.6 \mathrm{~Hz}\right), 134.2,129.5,129.3,128.5,127.8,127.6\left(\mathrm{~d},{ }^{3} J_{\mathrm{C}-\mathrm{F}}=8.0 \mathrm{~Hz}\right), 127.1,125.8,125.5$, $124.1\left(\mathrm{~d},{ }^{2} J_{\mathrm{C}-\mathrm{F}}=23.6 \mathrm{~Hz}\right), 123.9,120.9,117.2,111.4,111.1,109.9,62.4,44.5,35.4,21.5 \mathrm{ppm}$. HRMS (ESI): $m / z$ calcd. for $\mathrm{C}_{32} \mathrm{H}_{24} \mathrm{FN}_{2} \mathrm{O}_{4} \mathrm{~S}_{2}[\mathrm{M}+\mathrm{H}]^{+} 583.1156$, found 583.1161.

4-methyl-N-(2-((6-methyl-1-oxo-2-thiocyanato-2,3-dihydro-1H-inden-2-yl)(phenyl)methyl)benzofuran3-yl)benzenesulfonamide (3ac)

White solid; Flash column chromatography eluent (ethyl acetate/petroleum ether = 1:5); $15.3 \mathrm{mg}$ (66\% yield); m.p. $106-108{ }^{\circ} \mathrm{C}$. HPLC (Daicel Chiralpak IC, $n$-hexane/ethyl acetate $=70: 30$, flow rate $1.0 \mathrm{~mL} / \mathrm{min}$, detection at $254 \mathrm{~nm}): t_{\mathrm{R}}=9.2 \mathrm{~min}($ minor $), t_{\mathrm{R}}=11.2 \mathrm{~min}$ (major); 87:13 er. $[\alpha]_{\mathrm{D}}{ }^{25}=+100.7^{\circ}\left(\right.$ c $\left.0.47, \mathrm{CH}_{2} \mathrm{Cl}_{2}\right) .{ }^{1} \mathrm{H} \mathrm{NMR}\left(400 \mathrm{MHz}, \mathrm{CDCl}_{3}\right): \delta 7.73(\mathrm{~d}$, $J=7.6 \mathrm{~Hz}, 1 \mathrm{H}, \mathrm{ArH}), 7.55(\mathrm{~d}, J=8.4 \mathrm{~Hz}, 2 \mathrm{H}, \mathrm{ArH}), 7.46(\mathrm{~d}, J=8.0 \mathrm{~Hz}, 2 \mathrm{H}, \mathrm{ArH}), 7.41-7.30$ $(\mathrm{m}, 3 \mathrm{H}, \mathrm{ArH}), 7.19(\mathrm{~d}, J=8.0 \mathrm{~Hz}, 1 \mathrm{H}, \mathrm{ArH}), 7.10-7.00(\mathrm{~m}, 5 \mathrm{H}, \mathrm{ArH}), 6.86(\mathrm{~d}, J=8.4 \mathrm{~Hz}, 2 \mathrm{H}$, ArH), 6.70 (s, 1H, NH), $5.02(\mathrm{~s}, 1 \mathrm{H}, \mathrm{CH}), 3.94$ (d, J = $\left.18.8 \mathrm{~Hz}, 1 \mathrm{H}, \mathrm{CH}_{2}\right), 3.16$ (d, J = 18.8 Hz, $\left.1 \mathrm{H}, \mathrm{CH}_{2}\right), 2.35\left(\mathrm{~s}, 3 \mathrm{H}, \mathrm{CH}_{3}\right), 2.21\left(\mathrm{~s}, 3 \mathrm{H}, \mathrm{CH}_{3}\right)$ ppm. ${ }^{13} \mathrm{C} \mathrm{NMR}\left(100 \mathrm{MHz} \mathrm{CDCl}_{3}\right): \delta 198.4$, 153.7, 151.5, 147.1, 143.4, 138.7, 137.6, 136.0, 134.5, 133.1, 129.43, 129.36, 128.4, 127.6, 127.0, $126.0,125.8,125.41,125.38,123.9,121.0,117.0,111.3,110.3,62.0,44.3,35.5,21.5,21.0 \mathrm{ppm}$. HRMS (ESI): $m / z$ calcd. for $\mathrm{C}_{33} \mathrm{H}_{27} \mathrm{~N}_{2} \mathrm{O}_{4} \mathrm{~S}_{2}[\mathrm{M}+\mathrm{H}]^{+} 579.1407$, found 579.1396 .

N-(2-((6-bromo-1-oxo-2-thiocyanato-2,3-dihydro-1H-inden-2-yl)(phenyl)methyl)benzofuran-3-yl)4-methylbenzenesulfonamide (3ad)

White solid; Flash column chromatography eluent (ethyl acetate/petroleum ether = 1:5); $13.6 \mathrm{mg}$ (53\% yield); m.p. 198-200 ${ }^{\circ} \mathrm{C}$. HPLC (Daicel Chiralpak IC, $n$-hexane/ethyl acetate $=80: 20$, flow rate $1.0 \mathrm{~mL} / \mathrm{min}$, detection at $254 \mathrm{~nm}): t_{\mathrm{R}}=20.7 \mathrm{~min}($ minor $), t_{\mathrm{R}}=22.9 \mathrm{~min}$ (major); 70:30 er. $\left.[\alpha]_{\mathrm{D}}{ }^{25}=+35.5^{\circ}(c) .45, \mathrm{CH}_{2} \mathrm{Cl}_{2}\right) .{ }^{1} \mathrm{H} \mathrm{NMR}\left(400 \mathrm{MHz}, \mathrm{CDCl}_{3}\right): \delta 7.89$ (s, $1 \mathrm{H}, \mathrm{ArH}), 7.69-7.63(\mathrm{~m}, 2 \mathrm{H}, \mathrm{ArH}), 7.54(\mathrm{~d}, J=8.0 \mathrm{~Hz}, 1 \mathrm{H}, \mathrm{ArH}), 7.46(\mathrm{~d}, J=8.4 \mathrm{~Hz}, 1 \mathrm{H}$, ArH), 7.39 (t, $J=7.8 \mathrm{~Hz}, 1 \mathrm{H}, \mathrm{ArH}), 7.31(\mathrm{t}, J=7.6 \mathrm{~Hz}, 1 \mathrm{H}, \mathrm{ArH}), 7.20(\mathrm{~d}, J=8.0 \mathrm{~Hz}, 1 \mathrm{H}, \mathrm{ArH})$, 7.13-7.00 (m, 5H, ArH), $6.88(\mathrm{~d}, J=8.0 \mathrm{~Hz}, 1 \mathrm{H}, \mathrm{ArH}), 6.69$ (s, 1H, NH), $5.04(\mathrm{~s}, 1 \mathrm{H}, \mathrm{CH})$, $3.96\left(\mathrm{~d}, J=19.2 \mathrm{~Hz}, 1 \mathrm{H}, \mathrm{CH}_{2}\right), 3.19\left(\mathrm{~d}, J=19.2 \mathrm{~Hz}, 1 \mathrm{H}, \mathrm{CH}_{2}\right), 2.22\left(\mathrm{~s}, 3 \mathrm{H}, \mathrm{CH}_{3}\right) \mathrm{ppm} .{ }^{13} \mathrm{C}$ NMR $\left(100 \mathrm{MHz}_{,} \mathrm{CDCl}_{3}\right): \delta$ 197.1, 153.7, 151.2, 148.3, 143.5, 139.1, 136.0, 134.8, 134.2, 129.5, 129.3, 128.6, 128.3, 127.8, 127.6, 127.1, 125.9, 125.5, 123.9, 122.7, 121.0, 117.2, 111.4, 109.8, 62.0, 44.4, 35.6, 21.5 ppm. HRMS (ESI): $m / z$ calcd. for $\mathrm{C}_{32} \mathrm{H}_{24}{ }^{79} \mathrm{BrN}_{2} \mathrm{O}_{4} \mathrm{~S}_{2}[\mathrm{M}+\mathrm{H}]^{+} 643.0355$, found 643.0361; calcd. for $\mathrm{C}_{32} \mathrm{H}_{24}{ }^{81} \mathrm{BrN}_{2} \mathrm{O}_{4} \mathrm{~S}_{2}[\mathrm{M}+\mathrm{H}]^{+}$645.0335, found 645.0345.

N-(2-((6-methyoxy-1-oxo-2-thiocyanato-2,3-dihydro-1H-inden-2-yl)(phenyl)methyl)benzofuran-3yl)-4-methylbenzenesulfonamide (3ae)

White solid; Flash column chromatography eluent (ethyl acetate/petroleum ether = 1:5); $10.2 \mathrm{mg}$ (43\% yield); m.p. 118-120 ${ }^{\circ} \mathrm{C}$. HPLC (Daicel Chiralpak IA, n-hexane/ethyl acetate $=85: 15$, flow rate $1.0 \mathrm{~mL} / \mathrm{min}$, detection at $254 \mathrm{~nm}): t_{R}=43.3 \mathrm{~min}($ minor $), t_{R}=67.2 \mathrm{~min}$ (major); 86:14 er. $[\alpha]_{\mathrm{D}}{ }^{25}=+105.7^{\circ}\left(c\right.$ 1.46, $\left.\mathrm{CH}_{2} \mathrm{Cl}_{2}\right) .{ }^{1} \mathrm{H} \mathrm{NMR}\left(400 \mathrm{MHz}, \mathrm{CDCl}_{3}\right): \delta 7.71$ $(\mathrm{d}, J=8.0 \mathrm{~Hz}, 1 \mathrm{H}, \mathrm{ArH}), 7.55(\mathrm{~d}, J=8.4 \mathrm{~Hz}, 1 \mathrm{H}, \mathrm{ArH}), 7.47-7.45(\mathrm{~m}, 2 \mathrm{H}, \mathrm{ArH}), 7.38$ (td, $\left.J_{1}=7.6 \mathrm{~Hz}, J_{2}=1.2 \mathrm{~Hz}, 1 \mathrm{H}, \mathrm{ArH}\right), 7.32-7.29(\mathrm{~m}, 1 \mathrm{H}, \mathrm{ArH}), 7.207 .17(\mathrm{~m}, 2 \mathrm{H}, \mathrm{ArH}), 7.14-7.01$ $(\mathrm{m}, 6 \mathrm{H}, \mathrm{ArH}), 6.85(\mathrm{~d}, J=8.0 \mathrm{~Hz}, 1 \mathrm{H}, \mathrm{ArH}), 6.78(\mathrm{~s}, 1 \mathrm{H}, \mathrm{NH}), 5.05(\mathrm{~s}, 1 \mathrm{H}, \mathrm{CH}), 3.94(\mathrm{~d}$, $\left.J=18.8 \mathrm{~Hz}, 1 \mathrm{H}, \mathrm{CH}_{2}\right), 3.80\left(\mathrm{~s}, 3 \mathrm{H}, \mathrm{CH}_{3}\right), 3.14\left(\mathrm{~d}, J=18.4 \mathrm{~Hz}, 1 \mathrm{H}, \mathrm{CH}_{2}\right), 2.20\left(\mathrm{~s}, 3 \mathrm{H}, \mathrm{CH}_{3}\right)$ ppm. ${ }^{13} \mathrm{C}$ NMR $\left(100 \mathrm{MHz}, \mathrm{CDCl}_{3}\right): \delta 198.4,160.0,153.7,151.4,143.4,142.6,135.9,134.4$, $134.2,129.4,129.3,128.4,127.6,127.0,126.8,125.9,125.8,125.4,123.8,121.0,117.0,111.3$, $110.3,106.3,62.4,55.6,44.4,35.2,21.5 \mathrm{ppm}$. HRMS (ESI): $\mathrm{m} / z$ calcd. for $\mathrm{C}_{33} \mathrm{H}_{27} \mathrm{~N}_{2} \mathrm{O}_{5} \mathrm{~S}_{2}[\mathrm{M}$ $+\mathrm{H}]^{+}$595.1356, found 595.1347 . 
N-(2-((5-fluoro-1-oxo-2-thiocyanato-2,3-dihydro-1H-inden-2-yl)(phenyl)methyl)benzofuran-3-yl)4-methylbenzenesulfonamide (3af)

White solid; Flash column chromatography eluent (ethyl acetate/petroleum ether = 1:5); $10.5 \mathrm{mg}$ (45\% yield); m.p. $113-115^{\circ} \mathrm{C}$. HPLC (Daicel Chiralpak IA, $n$-hexane/ethyl acetate $=85: 15$, flow rate $1.0 \mathrm{~mL} / \mathrm{min}$, detection at $254 \mathrm{~nm}): t_{\mathrm{R}}=42.1 \mathrm{~min}$ (minor), $t_{\mathrm{R}}=48.7 \mathrm{~min}$ (major); $74: 26$ er. $[\alpha]_{\mathrm{D}}{ }^{25}=+65.8^{\circ}\left(\right.$ c $\left.1.2, \mathrm{CH}_{2} \mathrm{Cl}_{2}\right) .{ }^{1} \mathrm{H} \mathrm{NMR}\left(400 \mathrm{MHz}, \mathrm{CDCl}_{3}\right): \delta 7.78$ (dd, $\left.J_{1}=8.4 \mathrm{~Hz}, J_{2}=5.2 \mathrm{~Hz}, 1 \mathrm{H}, \mathrm{ArH}\right), 7.68(\mathrm{~d}, J=7.6 \mathrm{~Hz}, 1 \mathrm{H}, \mathrm{ArH}), 7.55(\mathrm{~d}, J=8.4 \mathrm{~Hz}, 1 \mathrm{H}$, $\mathrm{ArH}), 7.46(\mathrm{~d}, J=8.0 \mathrm{~Hz}, 2 \mathrm{H}, \mathrm{ArH}), 7.39\left(\mathrm{td}, J_{1}=7.8 \mathrm{~Hz}, J_{2}=1.2 \mathrm{~Hz}, 1 \mathrm{H}, \mathrm{ArH}\right), 7.32-7.29$ (m, 1H, ArH), $7.11-7.02(\mathrm{~m}, 6 \mathrm{H}, \mathrm{ArH}), 6.87(\mathrm{~d}, J=8.0 \mathrm{~Hz}, 2 \mathrm{H}, \mathrm{ArH}), 6.71(\mathrm{~s}, 1 \mathrm{H}, \mathrm{NH})$, $5.05(\mathrm{~s}, 1 \mathrm{H}, \mathrm{CH}), 4.03\left(\mathrm{~d}, J=19.2 \mathrm{~Hz}, 1 \mathrm{H}, \mathrm{CH}_{2}\right), 3.25\left(\mathrm{~d}, J=19.2 \mathrm{~Hz}, 1 \mathrm{H}, \mathrm{CH}_{2}\right), 2.21(\mathrm{~s}, 3 \mathrm{H}$, $\left.\mathrm{CH}_{3}\right)$ ppm. ${ }^{13} \mathrm{C} \mathrm{NMR}\left(100 \mathrm{MHz} \mathrm{CDCl}_{3}\right): \delta 196.7,167.9\left(\mathrm{~d},{ }^{1} J_{\mathrm{C}-\mathrm{F}}=258.3 \mathrm{~Hz}\right), 153.7,152.8$ $\left(d,{ }^{3} J_{C-F}=10.6 \mathrm{~Hz}\right), 151.2,143.5,135.9,134.2,129.4,129.3,128.5,128.0\left(d,{ }^{3} J_{C-F}=10.6 \mathrm{~Hz}\right)$, $127.8,127.1,125.8,125.5,123.9,120.9,117.2,117.0,113.0\left(\mathrm{~d},{ }^{2} J_{\mathrm{C}-\mathrm{F}}=22.9 \mathrm{~Hz}\right), 111.4,109.9$, 62.0, 44.5, 35.9, 21.5 ppm. HRMS (ESI): $m / z$ calcd. for $\mathrm{C}_{32} \mathrm{H}_{24} \mathrm{FN}_{2} \mathrm{O}_{4} \mathrm{~S}_{2}[\mathrm{M}+\mathrm{H}]^{+} 583.1156$, found 583.1148 .

N-(2-((5-chloro-1-oxo-2-thiocyanato-2,3-dihydro-1H-inden-2-yl)(phenyl)methyl)benzofuran-3-yl)4-methylbenzenesulfonamide (3ag)

White solid; Flash column chromatography eluent (ethyl acetate/petroleum ether = 1:5); $14.6 \mathrm{mg}\left(61 \%\right.$ yield); m.p. $128-130{ }^{\circ} \mathrm{C}$. HPLC (Daicel Chiralpak IA, $n$-hexane/2propanol $=90: 10$, flow rate $1.0 \mathrm{~mL} / \mathrm{min}$, detection at $254 \mathrm{~nm}$ ): $t_{\mathrm{R}}=42.9 \mathrm{~min}$ (minor), $t_{\mathrm{R}}=67.4 \mathrm{~min}$ (major); 62:38 er. $[\alpha]_{\mathrm{D}}{ }^{25}=+24.0^{\circ}\left(c 0.56, \mathrm{CH}_{2} \mathrm{Cl}_{2}\right) .{ }^{1} \mathrm{H}$ NMR $(400 \mathrm{MHz}$, $\left.\mathrm{CDCl}_{3}\right): \delta 7.69(\mathrm{t}, J=8.0 \mathrm{~Hz}, 2 \mathrm{H}, \mathrm{ArH}), 7.55(\mathrm{~d}, J=8.0 \mathrm{~Hz}, 1 \mathrm{H}, \mathrm{ArH}), 7.46(\mathrm{~d}, J=8.4 \mathrm{~Hz}$, 2H, ArH), 7.41-7.37 (m, 1H, ArH), 7.34-7.30 (m, 3H, ArH), 7.12-7.00 (m, 6H, ArH), 6.87 $(\mathrm{d}, J=8.0 \mathrm{~Hz}, 2 \mathrm{H}, \mathrm{ArH}), 6.63(\mathrm{~s}, 1 \mathrm{H}, \mathrm{NH}), 5.04(\mathrm{~s}, 1 \mathrm{H}, \mathrm{CH}), 4.00\left(\mathrm{~d}, J=19.2 \mathrm{~Hz}, 1 \mathrm{H}, \mathrm{CH}_{2}\right)$, $3.21\left(\mathrm{~d}, J=19.2 \mathrm{~Hz}, 1 \mathrm{H}, \mathrm{CH}_{2}\right), 2.22\left(\mathrm{~s}, 3 \mathrm{H}, \mathrm{CH}_{3}\right)$ ppm. ${ }^{13} \mathrm{C} \mathrm{NMR}\left(100 \mathrm{MHz}, \mathrm{CDCl}_{3}\right): \delta 197.1$, 153.7, 151.23, 151.15, 143.5, 143.0, 136.0, 134.2, 131.5, 129.5, 129.3, 128.6, 127.8, 127.1, 126.6, $126.4,125.9,125.5,123.9,121.0,117.2,111.4,109.8,61.8,44.4,35.6,21.6$ ppm. HRMS (ESI): $m / z$ calcd. for $\mathrm{C}_{32} \mathrm{H}_{24} \mathrm{ClN}_{2} \mathrm{O}_{4} \mathrm{~S}_{2}[\mathrm{M}+\mathrm{H}]^{+}$599.0861, found 599.0851.

N-(2-((5-bromo-1-oxo-2-thiocyanato-2,3-dihydro-1H-inden-2-yl)(phenyl)methyl)benzofuran-3-yl)4-methylbenzenesulfonamide (3ah)

White solid; Flash column chromatography eluent (ethyl acetate/petroleum ether = 1:5); $16.5 \mathrm{mg}$ (64\% yield); m.p. 106-108 ${ }^{\circ} \mathrm{C}$. HPLC (Daicel Chiralpak IA, $n$-hexane/ethyl acetate $=70: 30$, flow rate $1.0 \mathrm{~mL} / \mathrm{min}$, detection at $254 \mathrm{~nm}): t_{\mathrm{R}}=26.7 \mathrm{~min}($ minor $), t_{\mathrm{R}}=39.4 \mathrm{~min}$ (major); 69:31 er. $[\alpha]_{\mathrm{D}}{ }^{25}=+44.8^{\circ}\left(\right.$ c $\left.0.88, \mathrm{CH}_{2} \mathrm{Cl}_{2}\right) .{ }^{1} \mathrm{H} \mathrm{NMR}\left(400 \mathrm{MHz}, \mathrm{CDCl}_{3}\right): \delta 7.67(\mathrm{~d}$, $J=7.6 \mathrm{~Hz}, 1 \mathrm{H}, \mathrm{ArH}), 7.62(\mathrm{~d}, J=8.4 \mathrm{~Hz}, 1 \mathrm{H}, \mathrm{ArH}), 7.55(\mathrm{~d}, J=8.4 \mathrm{~Hz}, 1 \mathrm{H}, \mathrm{ArH}), 7.50-7.45$ $(\mathrm{m}, 4 \mathrm{H}, \mathrm{ArH}), 7.39\left(\mathrm{td}, J_{1}=7.6 \mathrm{~Hz}, J_{2}=1.2 \mathrm{~Hz}, 1 \mathrm{H}, \mathrm{ArH}\right), 7.33-7.29(\mathrm{~m}, 1 \mathrm{H}, \mathrm{ArH}), 7.12-7.01$ $(\mathrm{m}, 5 \mathrm{H}, \mathrm{ArH}), 6.87(\mathrm{~d}, J=8.4 \mathrm{~Hz}, 2 \mathrm{H}, \mathrm{ArH}), 6.68(\mathrm{~s}, 1 \mathrm{H}, \mathrm{NH}), 5.05(\mathrm{~s}, 1 \mathrm{H}, \mathrm{CH}), 4.01(\mathrm{~d}$, $\left.J=19.2 \mathrm{~Hz}, 1 \mathrm{H}, \mathrm{CH}_{2}\right), 3.22\left(\mathrm{~d}, J=18.8 \mathrm{~Hz}, 1 \mathrm{H}, \mathrm{CH}_{2}\right), 2.21\left(\mathrm{~s}, 3 \mathrm{H}, \mathrm{CH}_{3}\right) \mathrm{ppm} .{ }^{13} \mathrm{C} \mathrm{NMR}(100$ $\left.\mathrm{MHz}_{2} \mathrm{CDCl}_{3}\right): \delta 197.3,153.7,151.2,151.1,143.5,135.9,134.2,132.3,132.0131 .9,129.4,129.3$, 128.6, 127.8, 127.1, 126.6, 125.8, 125.5, 123.9, 120.9, 117.2, 111.4, 109.8, 61.8, 44.4, 35.6, 21.5 ppm. HRMS (ESI): $m / z$ calcd. for $\mathrm{C}_{32} \mathrm{H}_{24}{ }^{79} \mathrm{BrN}_{2} \mathrm{O}_{4} \mathrm{~S}_{2}[\mathrm{M}+\mathrm{H}]^{+}$643.0355, found 643.0359; calcd. for $\mathrm{C}_{32} \mathrm{H}_{24}{ }^{81} \mathrm{BrN}_{2} \mathrm{O}_{4} \mathrm{~S}_{2}[\mathrm{M}+\mathrm{H}]^{+}$645.0335, found 645.0342.

4-methyl-N-(2-((1-oxo-5-(oxo-l6-methyl)-2-thiocyanato-2,3-dihydro-1H-inden-2-yl)(phenyl)meth yl)benzofuran-3-yl)benzenesulfonamide (3ai)

White solid; Flash column chromatography eluent (ethyl acetate/petroleum ether $=1: 5$ ); $15.2 \mathrm{mg}$ (64\% yield); m.p. 103-105 ${ }^{\circ} \mathrm{C}$. HPLC (Daicel Chiralpak IA, $n$-hexane/ethyl acetate $=70: 30$, flow rate $1.0 \mathrm{~mL} / \mathrm{min}$, detection at $254 \mathrm{~nm}): t_{\mathrm{R}}=10.1 \mathrm{~min}($ minor $), t_{\mathrm{R}}=12.7 \mathrm{~min}$ (major); 85:15 er. $[\alpha]_{\mathrm{D}}{ }^{25}=+78.6^{\circ}\left(\mathrm{c} 1.12, \mathrm{CH}_{2} \mathrm{Cl}_{2}\right) .{ }^{1} \mathrm{H} \mathrm{NMR}\left(400 \mathrm{MHz}, \mathrm{CDCl}_{3}\right): \delta 7.74(\mathrm{~d}$, $J=7.2 \mathrm{~Hz}, 1 \mathrm{H}, \mathrm{ArH}), 7.68(\mathrm{~d}, J=8.4 \mathrm{~Hz}, 1 \mathrm{H}, \mathrm{ArH}), 7.54(\mathrm{~d}, J=8.4 \mathrm{~Hz}, 1 \mathrm{H}, \mathrm{ArH}), 7.46(\mathrm{~d}$, $J=8.4 \mathrm{~Hz}, 2 \mathrm{H}, \mathrm{ArH}), 7.41-7.36(\mathrm{~m}, 1 \mathrm{H}, \mathrm{ArH}), 7.36-7.30$ (m, 1H, ArH), 7.10-7.02 (m, 5H, ArH), 6.87-6.84 (m, 3H, ArH), 6.75-6.71 (m, 2H, ArH + NH), $5.00(\mathrm{~s}, 1 \mathrm{H}, \mathrm{CH}), 3.96(\mathrm{~d}$, 
$\left.J=18.8 \mathrm{~Hz}, 1 \mathrm{H}, \mathrm{CH}_{2}\right), 3.81\left(\mathrm{~s}, 3 \mathrm{H}, \mathrm{CH}_{3}\right), 3.19\left(\mathrm{~d}, J=18.8 \mathrm{~Hz}, 1 \mathrm{H}, \mathrm{CH}_{2}\right), 2.20\left(\mathrm{~s}, 3 \mathrm{H}, \mathrm{CH}_{3}\right)$ ppm. ${ }^{13} \mathrm{C}$ NMR $\left(100 \mathrm{MHz}, \mathrm{CDCl}_{3}\right): \delta 196.5,166.5,153.7,153.0,151.4,143.4,135.9,134.5$, $129.4,129.3,128.4,127.6,127.3,127.0,126.1,126.0,125.4,123.8,121.0,117.1,116.6,111.3$, $110.4,109.3,62.3,55.7,44.5,35.9,21.5$ ppm. HRMS (ESI): $m / z$ calcd. for $\mathrm{C}_{33} \mathrm{H}_{27} \mathrm{~N}_{2} \mathrm{O}_{5} \mathrm{~S}_{2}[\mathrm{M}$ $+\mathrm{H}]^{+}$595.1356, found 595.1364 .

Supplementary Materials: Spectroscopic data $\left({ }^{1} \mathrm{H}\right.$ and ${ }^{13} \mathrm{C}$ NMR) and chiral HPLC chromatograms for all new compounds 3 .

Author Contributions: X.-Y.D. performed the experiments, acquired and analyzed the original data, and wrote the preliminary manuscript. D.-M.D. conceived and designed the experiments, revised all figures and schemes, analyzed the data, and reviewed and edited the manuscript. All authors have read and agreed to the published version of the manuscript.

Funding: This research received no external funding.

Institutional Review Board Statement: Not applicable.

Informed Consent Statement: Not applicable.

Data Availability Statement: Not applicable.

Conflicts of Interest: The authors declare no conflict of interest.

Sample Availability: Samples of the compounds 3 are available from the authors.

\section{References}

1. Dawood, K.M. Benzofuran derivatives: A patent review. Expert Opin. Ther. Pat. 2013, 23, 1133-1156. [CrossRef]

2. Radadiya, A.; Shah, A. Bioactive benzofuran derivatives: An insight on lead developments, radioligands and advances of the last decade. Eur. J. Med. Chem. 2015, 97, 356-376. [CrossRef]

3. Masubuchi, M.; Kawasaki, K.; Ebiike, H.; Ikeda, Y.; Tsujii, S.; Sogabe, S.; Fujii, T.; Sakata, K.; Shiratori, Y.; Aoki, Y.; et al. Design and synthesis of novel benzofurans as new class of antifungal agents targeting fungal N-myristoyltransferase. Part 1 . Bioorg. Med. Chem. Lett. 2001, 11, 1833-1837. [CrossRef]

4. Nevagi, R.J.; Dighe, S.N. Biological and medicinal significance of benzofuran. Eur. J. Med. Chem. 2015, 97, 561-581. [CrossRef]

5. Hiremathad, A.; Patil, M.R.; Chethana, K.R.; Chand, K.; Santos, M.A.; Keri, R.S. Benzofuran: An emerging scaffold for antimicrobial agents. RSC Adv. 2015, 5, 96809-96828. [CrossRef]

6. Mitsui, C.; Soeda, J.; Miwa, K.; Tsuji, H.; Takeya, J.; Nakamura, E. Naphtho[2,1-b:6,5-b']difuran: A versatile motif available for solution-processed single-crystal organic field-effect transistors with high hole mobility. J. Am. Chem. Soc. 2012, 134, 5448-5451. [CrossRef] [PubMed]

7. Bickoff, E.M.; Booth, A.N.; Lyman, R.L.; Livingston, A.L.; Thompson, C.R.; Deeds, F. Coumestrol, a new estrogen isolated from forage crops. Science 1958, 6, 536-539. [CrossRef]

8. Xu, Z.; Zhao, S.J.; Lv, Z.S.; Feng, L.S.; Wang, Y.L.; Zhang, F.; Bai, L.Y.; Deng, J.L. Benzofuran derivatives and their anti-tubercular, anti-bacterial activities. Eur. J. Med. Chem. 2019, 162, 266-276. [CrossRef] [PubMed]

9. Gu, Z.; Zhou, J.; Jiang, G.F.; Zhou, Y.G. Synthesis of chiral $\gamma$-aminophosphonates through the organocatalytic hydrophosphonylation of azadienes with phosphites. Org. Chem. Front. 2018, 5, 1148-1151. [CrossRef]

10. Gu, Z.; Xie, J.J.; Jiang, G.F.; Zhou, Y.G. Catalytic asymmetric conjugate addition of tritylthiol to azadienes with a bifunctional organocatalyst. Asian J. Org. Chem. 2018, 7, 1561-1564. [CrossRef]

11. Lin, W.; Zhang, C.; Xu, W.; Cheng, Y.Y.; Li, P.F.; Li, W.J. Organocatalytic asymmetric Michael addition of rhodanines to azadienes for assembling of sulfur-containing tetrasubstituted carbon stereocenters. Adv. Synth. Catal. 2019, 361, 476-480. [CrossRef]

12. Lin, W.; Lin, X.; Cheng, Y.Y.; Chang, X.Y.; Zhou, S.; Li, P.F.; Li, W.J. Organocatalytic enantioselective direct vinylogous Michael addition of $\gamma$-substituted deconjugate butenolides to azadienes. Org. Chem. Front. 2019, 6, 2452-2456. [CrossRef]

13. Li, S.F.; Zhang, C.; Cheng, Y.Y.; Jia, Q.F.; Li, W.J.; Liu, K.; Li, P.F. Enantioselective construction of vicinal sulfur-functionalized quaternary and tertiary stereocenters via organocatalytic Michael addition of 5H-Thiazol-4-ones to 1-azadienes. Asian J. Org. Chem. 2020, 9, 1183-1186. [CrossRef]

14. Yan, J.Z.; Li, X.P.; Chen, Y.Z.; Li, Y.; Chen, W.W.; Zhan, R.T.; Huang, H.C. Organocatalytic 1,4-addition of azadienes with 3-homoacyl coumarins toward highly enantioenriched benzofuran coumarin skeletons. J. Org. Chem. 2020, 85, 12175-12186. [CrossRef] [PubMed]

15. Brown, P.D.; Morra, M.J. Fate of ionic thiocyanate (SCN-) in soil. J. Agric. Food Chem. 1993, 41, 978-982. [CrossRef]

16. Garson, M.J.; Simpson, J.S. Marine isocyanides and related natural products-Structure, biosynthesis and ecology. Nat. Prod. Rep. 2004, 21, 164-179. [CrossRef]

17. Elhalem, E.; Bailey, B.N.; Docampo, R.; Ujváry, I.; Szajnman, B.H.; Rodriguez, J. Design, synthesis, and biological evaluation of aryloxyethyl thiocyanate derivatives against trypanosoma cruzi. J. Med. Chem. 2002, 45, 3984-3999. [CrossRef] 
18. Dutta, S.; Abe, H.; Aoyagi, S.; Kibayashi, C.; Gates, K.S. DNA Damage by fasicularin. J. Am. Chem. Soc. 2005, $127,15004-15005$. [CrossRef] [PubMed]

19. Yasman; Edrada, A.R.; Wray, V.; Proksch, P. New 9-thiocyanatopupukeanane sesquiterpenes from the nudibranch phyllidia varicosa and its sponge-prey axinyssa aculeate. J. Nat. Prod. 2003, 66, 1512-1514. [CrossRef] [PubMed]

20. Chandler, J.D.; Day, B.J. Thiocyanate: A potentially useful therapeutic agent with host defense and antioxidant properties. Biochem. Pharmacol. 2012, 84, 1381-1387. [CrossRef]

21. Zhu, N.; Wang, F.; Chen, P.H.; Ye, J.X.; Liu, G.S. Copper-catalyzed intermolecular trifluoromethylazidation and trifluoromethylthiocyanation of allenes: Efficient access to $\mathrm{CF}_{3}$-containing allyl azides and thiocyanates. Org. Lett. 2015, 17, 3580-3583. [CrossRef]

22. Chen, Q.; Lei, Y.J.; Wang, Y.F.; Wang, C.; Wang, Y.N.; Xu, Z.Q.; Wang, H.; Wang, R. Direct thiocyanation of ketene dithioacetals under transition-metal-free conditions. Org. Chem. Front. 2017, 4, 369-372. [CrossRef]

23. Yang, H.; Duan, X.H.; Zhao, J.F.; Guo, L.N. Transition-metal-free tandem radical thiocyanooxygenation of olefinic amides: A new route to SCN-containing heterocycles castanheiro. Org. Lett. 2015, 17, 1998-2001. [CrossRef] [PubMed]

24. Castanheiro, T.; Gulea, M.; Donnard, M.; Suffert, J. Practical access to aromatic thiocyanates by CuCN-mediated direct aerobic oxidative cyanation of thiophenols and diaryl disulfides. Eur. J. Org. Chem. 2014, 2014, 7814-7817. [CrossRef]

25. Zhu, D.; Chang, D.H.; Shi, L. Transition-metal-free cross-coupling of thioethers with aryl(cyano)iodonium triflates: A facile and efficient method for the one-pot synthesis of thiocyanates. Chem. Commun. 2015, 51, 7180-7183. [CrossRef]

26. Teng, F.; Yu, J.T.; Yang, H.T.; Jiang, Y.; Cheng, J. Copper-catalyzed cyanation of disulfides by azobisisobutyronitrile leading to thiocyanates. J. Chem. Commun. 2014, 50, 12139-12141. [CrossRef]

27. Xu, Q.; Zhang, L.Y.; Feng, G.F.; Jin, C.G. Progress on the synthesis and applications of thiocyanates. Chin. J. Org. Chem. 2019, 39, 287-300. [CrossRef]

28. Castanherio, T.; Suffert, J.; Donnard, M.; Gulea, M. Recent advances in the chemistry of organic thiocyanates. Chem. Soc. Rev. 2016, 45, 494-505. [CrossRef] [PubMed]

29. Yu, L.; Wu, X.Y.; Kim, J.M.; Vaithiyanathan, V.; Liu, Y.D.; Tan, Y.; Qin, W.L.; Song, C.E.; Yan, H.L. Asymmetric synthesis of 2-thiocyanato-2-(1-aminoalkyl)-substituted 1-tetralones and 1-indanones with tetrasubstituted carbon stereogenic centers via cooperative cation-binding catalysis. Adv. Synth. Catal. 2017, 359, 1879-1891. [CrossRef]

30. CCDC 2100823 Contains the Supplementary Crystallographic Data for This Paper. These Data Can Be Obtained Free of Charge via. Available online: http:/ /www.ccdc.cam.ac.uk/conts/retrieving.html (accessed on 2 August 2021).

31. Wang, C.S.; Li, T.Z.; Cheng, Y.C.; Zhou, J.; Mei, G.J.; Shi, F. Catalytic asymmetric [4 + 1] cyclization of benzofuran-derived azadienes with 3-chlorooxindoles. J. Org. Chem. 2019, 84, 3214-3222. [CrossRef] [PubMed]

32. Shi, Z.G.; Tong, Q.J.; Leong, W.W.Y.; Zhong, G.F. [4+2] Annulation of vinyl ketones initiated by a phosphine-catalyzed AzaRauhut-Currier reaction: A practical access to densely functionalized tetrahydropyridines. Chem. Eur. J. 2012, 18, $9802-9806$. [CrossRef] [PubMed]

33. Rong, Z.Q.; Wang, M.; Chow, C.H.E.; Zhao, Y. A catalyst-enabled diastereodivergent Aza-Diels-Alder reaction: Complementarity of N-Heterocyclic Carbenes and chiral amines. Chem. Eur. J. 2016, 22, 9483-9487. [CrossRef] [PubMed]

34. Zhu, Y.; Malerich, J.P.; Rawal, V.H. Squaramide-catalyzed enantioselective Michael addition of diphenyl phosphite to nitroalkenes. Angew. Chem. Int. Ed. 2010, 49, 153-156. [CrossRef] [PubMed]

35. Yang, W.; Du, D.M. Highly enantioselective Michael addition of nitroalkanes to chalcones using chiral squaramides as hydrogen bonding organocatalysts. Org. Lett. 2010, 12, 5450-5453. [CrossRef]

36. Yang, W.; Du, D.M. Chiral squaramide-catalyzaed highly enanioselective Michael addition of 2-hydroxy-1,4-naphthoquinones to nitroalkenes. Adv. Synth. Catal. 2011, 353, 1241-1246. [CrossRef]

37. Vakulya, B.; Varga, S.; Csampai, A.; Soós, T. Highly enantioselective conjugate addition of nitromethane to chalconesusing bifunctional cinchona organocatalysts. Org. Lett. 2005, 7, 1967-1969. [CrossRef] 